\title{
Plagl1 is part of the mammalian retinal injury response and a critical regulator of Müller glial cell quiescence
}

Short title: Plagll sustains Müller glia quiescence

Yacine Touahri ${ }^{1,2,3,4}$, Luke Ajay David ${ }^{1,5}$, Yaroslav Ilnytskyy ${ }^{6}$, Edwin van Oosten $^{1,2}$, Joseph Hanna $^{1,5}$, Nobuhiko Tachibana ${ }^{1,3}$, Lata Adnani ${ }^{1,3}$, Jiayi Zhao ${ }^{1,2}$, Mary Hoffman ${ }^{3}$, Rajiv Dixit ${ }^{1,2,3,4}$, Laurent Journot $^{7}$, Yves Sauve ${ }^{8}$, Igor Kovalchuk ${ }^{6}$, Isabelle Aubert ${ }^{1,5}$, Jeffrey Biernaskie ${ }^{4}$, Carol Schuurmans $1,2,3,5$

${ }^{1}$ Sunnybrook Research Institute, 2075 Bayview Ave, Toronto, ON, Canada, M4N 3M5

${ }^{2}$ Department of Biochemistry, University of Toronto, Toronto, ON, Canada

${ }^{3}$ Department of Biochemistry and Molecular Biology, Alberta Children's Hospital Research

Institute, Hotchkiss Brain Institute, University of Calgary, Calgary, AB, Canada

${ }^{4}$ Department of Comparative Biology and Experimental Medicine, Alberta Children's Hospital

Research Institute, Hotchkiss Brain Institute, University of Calgary, Calgary, AB, Canada

${ }^{5}$ Department of Laboratory Medicine and Pathobiology, University of Toronto, Toronto, ON,

Canada

${ }^{6}$ Dept of Biological Sciences, University of Lethbridge, Lethbridge, AB, Canada.

${ }^{7}$ Institut de Génomique Fonctionnelle, Montpellier, France

${ }^{8}$ Department of Ophthalmology and Visual Sciences and Department of Physiology, University

of Alberta, Edmonton, Alberta, Canada

*Corresponding author:

Carol Schuurmans (cschuurm@sri.utoronto.ca)

S116, Sunnybrook Research Institute

2075 Bayview Ave

Toronto, Ontario, Canada, M4N 3M5

Telephone: 416-480-6100 ext 3266

Keywords: Müller glia, reactive gliosis, proliferation, neurogenesis, transcriptomics, Notch signaling, imprinted gene 


\begin{abstract}
Retinal damage triggers reactive gliosis in Müller glia across vertebrate species, but only in regenerative animals, such as teleost fish, do Müller glia initiate repair; proliferating and undergoing neurogenesis to replace lost cells. By mining scRNA-seq and bulk RNA-seq datasets, we found that Plagl1, a maternally imprinted gene, is dynamically regulated in reactive Müller glia post-insult, with transcript levels transiently increasing before stably declining. To study Plagll retinal function, we examined Plagl1 ${ }^{+/ \text {pat }}$ null mutants postnatally, revealing defects in retinal architecture, visual signal processing and a reactive gliotic phenotype. Plagl ${ }^{+/ \text {pat }}$ Müller glia proliferate ectopically and give rise to inner retinal neurons and photoreceptors. Transcriptomic and ATAC-seq profiles revealed similarities between $\mathrm{Plagll}^{+/ \text {pat }}$ retinas and neurodegenerative and injury models, including an upregulation of pro-gliogenic and proproliferative pathways, such as Notch, not observed in wild-type retinas Plagll is thus an essential component of the transcriptional regulatory networks that retain mammalian Müller glia in quiescence.
\end{abstract}




\section{INTRODUCTION}

Sight begins when photosensitive retinal neurons, the rod and cone photoreceptors, detect and convert light to electrical signals that are propagated via retinal interneurons to ganglion cells, and onto visual processing centers in the brain. Visual impairment afflicts 2.2 billion people worldwide (World Health Organization, 2019) and nearly half of these ailments are untreatable. In regenerating tissues, somatic stem cells transit from quiescent to active states to maintain tissue homeostasis and repair damage. However, most of the mammalian central nervous system (CNS), including the retina, is devoid of active stem cells and dying neurons are not replaced ${ }^{1}$. Müller glia are post-mitotic, terminally differentiated somatic cells that have a host of functions under normal physiological conditions, including the provision of structural and nutrient support to other retinal cells, recycling of photopigments, and functioning as optical light fibers ${ }^{2,3}$. Strikingly, in teleost fish and Xenopus, Müller glia behave similar to somatic stem cells in regenerative tissues, responding to injury by first de-differentiating into proliferating progenitor cells that then acquire neurogenic competence to replace lost retinal cells ${ }^{4,5}$. In contrast, very few Müller glia re-enter the cell cycle in mammals in response to injury, and fewer yet undergo neurogenesis ${ }^{6}$.

A recent ground breaking study employing bulk RNA-seq, single cell (sc) RNA-seq and ATAC-seq in fish and mice in two injury models defined three Müller glial states, each upheld by distinct gene regulatory networks: resting, reactive and restoration to resting, the latter state specific to mouse ${ }^{7}$. A reactive Müller glial state is triggered by injury and in both mouse and fish involves rapid and slow response genes. In both fish and mice, rapid response genes mediate TNF, MAPK, NFאB and Hippo signaling, as well as ribosome biogenesis in fish, which instead are activated as a slow response in mouse ${ }^{7}$. The slow response genes in mouse involve the reversion to quiescence genes, whereas in fish, cell cycle genes are elevated, as are genes that confer neurogenic competence ${ }^{7}$.

The reactive state common to mouse and fish is known as Müller glial gliosis, a stressinduced neuroprotective response characterized by cellular hypertrophy and barrier formation around the injury site, as well as the secretion of neuronal pro-survival factors, antioxidants, and pro-endothelial molecules ${ }^{6}$. While moderate gliosis is transient and protective, severe gliosis is cytotoxic, and scars and remodels the retina permanently. Common features of reactive gliosis include increased expression of intermediate filament genes (e.g. GFAP, Nestin) and increased ERK signaling ${ }^{6}$. Other responses are stressor-specific, including reduced expression of 
Kcnj10/Kir4.1, a potassium channel, glutamate-ammonia ligase (Glul) (also known as glutamine synthetase), and Cdkn1b/p27Kip1, a cell cycle inhibitor ${ }^{6}$.

Post-injury, mammalian Müller glia halt in a reactive state and then revert to quiescence, without proceeding to the next stage in regeneration, which is proliferation, as proliferation cues are rate-limiting ${ }^{8}$. ERK signaling, which is activated downstream of growild-typeh factor stimulation, is initiated in Müller glia by insult in all species ${ }^{5}$, and blocking ERK prevents Müller glia proliferation in fish ${ }^{9}$, and the low transient proliferation seen in birds ${ }^{10}$ and to a lesser extent in mammals ${ }^{11,12}$. Several other signals can also induce mammalian Müller glia to proliferate when ectopically activated, including Wnt ${ }^{13,14}$, Notch ${ }^{13,14}$, and Hedgehog ${ }^{15}$. Neurogenesis cues are also rate-limiting in mammals. Lineage-specifying transcription factors act in a combinatorial fashion to specify the fates of each of the seven retinal cell types ${ }^{16}$. Several lineage-specifiers are upregulated post-injury in Müller glia in fish, including Ascl1 ${ }^{17,18}$, which is required for injuryinduced Müller glia neurogenesis in fish ${ }^{19}$, but is not induced and rate-limiting in mammalian Müller cells ${ }^{20,21}$. Ascll overexpression can induce neurogenesis when misexpressed in the early postnatal retina, or when combined with HDAC inhibitors, Ascll can also induce adult neurogenesis, primarily promoting a bipolar cell-like fate ${ }^{22}$.

Given the potential for endogenous repair based on observations in fish and frogs $\mathrm{s}^{4,5}$, the search is on for genes that prevent mammalian Müller glia from proceeding to the proliferative and neurogenic stages of regeneration. We focused our attention on Plagll, a maternally imprinted gene which, when overexpressed in the developing Xenopus ${ }^{23}$ or murine ${ }^{24,25}$ retina, can induce cell cycle exit. The mammalian genome contains 150 imprinted genes, each mono-allelically expressed in a parent-of-origin-specific manner due to selective methylation of the maternal or paternal allele. Interestingly, there is growing support for the idea that even though imprinted genes encode a wide array of molecules (ncRNAs, transcription factors, signaling molecules, etc. $)^{26}$, many have a common role in maintaining somatic stem cell quiescence, including in the lung, hematopoietic system, skeletal muscle, and brain ${ }^{27-30}$. Consistent with the idea that imprinted genes may have co-evolved to suit a common purpose, a meta-analyses of 85 imprinted genes revealed that they form subnetworks of co-regulated biallelically-expressed and imprinted genes ${ }^{27,31}$. Plagll is a maternally imprinted gene encoding a zinc finger transcription factor that is part of an imprinted gene network (IGN) of 13 tightly co-regulated imprinted genes ${ }^{31}$. Here we studied Plagll function in the postnatal retina, revealing functional, structural, transcriptomic and 
epigenetic changes that culminate in an essential role for Plagll in maintaining Müller glial quiescence.

\section{RESULTS}

\section{Plagl1 is expressed in Müller glial and is dynamically regulated in response to retinal damage}

To determine whether Plagll could function in differentiated Müller glia, we first examined transcript distribution in postnatal day (P) 7 mouse retinas, when neurogenesis and gliogenesis is complete centrally ${ }^{32}$. Using RNAscope in situ hybridization, Plagll transcripts were detected in the inner nuclear layer (INL) and ganglion cell layer (GCL), overlapping with mRNA puncta for Sox9 (Fig. 1a), a pan-glial marker expressed in Müller glia in the INL and astrocytes in the $\mathrm{GCL}^{33}$. To assess Plagll expression at adult stages, we mined a published RNA-seq data set acquired from GFP-enriched Müller glia sorted from 2 month old GLASTCreERT2;Sun1-sGFP mice ${ }^{7}$. We found that Plagll transcript levels were enriched in $\mathrm{GFP}^{+}$cells at comparable levels to other Müller glia markers (e.g., Sox9, Vim, Sox2, Glul, Rlbp), contrasting to genes marking other retinal cell types that were instead enriched in GFP- cells (e.g., Vsx1, Vsx2, Crx, Otx2) (Fig. 1b). Plagll is thus expressed in murine Müller glia from early postnatal to adult stages.

In fish and frogs, Müller glia behave like somatic stem cells, regenerating all retinal cell types to promote repair ${ }^{4}$. Conversely, mammalian Müller glia respond to insult by undergoing reactive gliosis, a stress-induced neuroprotective response characterized by cellular hypertrophy and barrier formation around the injury site ${ }^{3}$. To determine how Plagll expression levels change in response to injury, we first mined the comprehensive suite of scRNA-seq and bulk RNA-seq data sets performed on enriched pools of two-month-old Müller glia following NMDA and light damage (LD) injuries ${ }^{7}$. At the single cell level, in accordance with our RNAscope analysis, Plagll was expressed in resting Müller glia and in astrocytes (Fig. 1c). However, Plagll transcripts were comparatively higher in activated Müller glia, indicative of an up-regulation following retinal damage. To better characterise the dynamics of Plagll expression in response to injury, we examined the distribution of Plagll transcripts in bulk RNA-seq data collected from Müller glia at 3-, 6-, and 12-, and 36-hr following NMDA treatment, and at 4-, 16-, and 24-hr post LD 7 . Plagll transcript levels rapidly escalated post-injury, within $3 \mathrm{hr}$ after NMDA treatment and $4 \mathrm{hr}$ after LD (Fig. 1d). However, very rapidly soon after, Plagll transcript levels declined below baseline, by 12-hr post-NMDA and within 16-hr post-LD (Fig. 1d). These data suggest that Plagll has a 
dynamic response to injury, with its downregulation coinciding with the switch of Müller glia from a resting to reactive state.

To further characterise Plagll expression following retinal damage, we performed our own injury, injecting 2 mo CD1 mice with N-methyl-N-nitrosourea (MNU), which rapidly induces photoreceptor degeneration ${ }^{34}$. First, we confirmed the degenerative effects of MNU after 3-, 7and 21-days post- treatment, revealing that GFAP was strongly upregulated at all stages, at the protein and transcript level, a sign that the reactive gliotic state has been triggered and is sustained (Fig. 1e, Supplementary Fig. ${ }^{1 \mathrm{a}-\mathrm{b}}$ ). In addition, we confirmed a partial photoreceptor loss at day 3 post-MNU, that was complete at days 7 and 21, as revealed by reduced expression of Rhodopsin and Rcvrn, rod markers, as well as Arr3, a cone marker (Fig. 1e, Supplementary Fig. ${ }^{1 \mathrm{a}-\mathrm{b}}$ ). To assess the effects of MNU on Plagll expression, we first performed Plagl1/Sox9 RNAscope, revealing an apparent decline in Plagll transcript levels compared to Sox9 (Fig. 1e, Supplementary Fig. ${ }^{1 \mathrm{a}-\mathrm{b}}$ ). To confirm these findings, and to further stratify the response period, we collected retinas at 3-, 6-, 12-, 24- and 72-hr and 7- and 21-days post-MNU and performed qPCR to analyse Gfap and Plagll transcript levels (Fig. 1f, Supplementary Fig. ${ }^{1 \mathrm{c}-\mathrm{d}}$ ). Plagll transcript levels increased within 3 hr-post MNU treatment, even before Gfap levels increased, and remained above baseline until $72 \mathrm{hr}$, when they began to decline, remaining below baseline up to 21-days post-injury (Fig. 1f, Supplementary Fig. ${ }^{1 \mathrm{c}-\mathrm{d}}$ ). Strikingly, the decline in Plagll transcript levels coincides with the transitory proliferation that occurs between $48-$ to $60-\mathrm{hr}$ post-MNU ${ }^{35}$. This dynamic expression profile, especially the correlation between Plagll decline and Müller glia activation and proliferation, prompted us to question whether Plagll had a role in controlling Müller glia function using a loss-of-function approach.

\section{Plagl1 is required to maintain retinal integrity and proper visual function}

Plagll is a maternally imprinted gene, resulting in mono-allelic expression from the paternal allele ${ }^{36}$. To study Plagll 'loss of function' mutants, we generated Plagl1 ${ }^{+/ \text {pat }}$ mice carrying a silenced maternal allele and mutant paternal allele, these mice were previously confirmed to lack Plagll expression in various tissues, including the embryonic retina ${ }^{24,27,31}$. While $80 \%$ of $\mathrm{Plagl1}^{+/ \text {pat }}$ mice die in the early perinatal period ${ }^{24,31}$, we were able to collect surviving Plagl1 ${ }^{+/- \text {pat }}$ animals at perinatal (P7), juvenile (P14, P21) and early adulthood (P60) stages. We first performed a morphological/structural assessment of the outer nuclear layer (ONL), 
INL and GCL using optical coherence tomography (OCT) in live P21 animals (Fig. 2a). In contrast to P21 wild-type controls, OCT images of Plagl1 ${ }^{+/ \text {pat }}$ retinas revealed that layer borders were less distinct, with hypo-reflective puncta in the inner plexiform layer (ipl), indicative of layer disruption in focal areas of the retina (Fig. 2a). In transverse sections through the retina, DAPI nuclear staining confirmed these OCT observations and revealed striking retinal dysmorphologies in Plagl1 $1^{+/ \text {pat }}$ retinas at all postnatal stages assessed (P7, P14, P21, P60), which became increasingly prevalent over time (Fig. 2b-c). Two distinct abnormalities were observed; ectopias, defined as protrusions of ONL nuclei into the subretinal space, and rosettes, defined as focal dysplasias that formed in the ONL but protruded into, and disformed, the INL (Fig. 2b, Supplementary Fig. ${ }^{2 a-c}$ ).

To assess these retinal abnormalities further, we immunostained P21 wild-type and Plagl1 ${ }^{+/ \text {pat }}$ retinas with molecular markers for the seven retinal cell types. The most striking disruptions were observed in the ONL, especially in the positioning of rod ( Rhodospin $^{+}$) and cone $\left(\mathrm{Arr}^{+}, \mathrm{m}\right.$-opsin $\left.{ }^{+}, \mathrm{s}-\mathrm{opsin}{ }^{+}\right)$outer segments, which were internalized in the central lumens of the Plagl1 ${ }^{+/ \text {pat }}$ retinal rosettes (Fig. 2d, Supplementary Fig. ${ }^{3 \mathrm{a}-\mathrm{b}}$ ). Of the remaining cell types, amacrine $\left(\mathrm{Pax}^{+}\right.$, calretinin ${ }^{+}$, syntaxin $\left.^{+}\right)$, bipolar $\left(\mathrm{Chx} 10^{+}, \mathrm{PKC}^{+}\right)$, horizontal (calbindin $\left.{ }^{+}\right)$, and ganglion


in the vicinity of the Plagl1 ${ }^{+/ \text {-pat }}$ rosettes (Supplementary Fig. ${ }^{3 c-j}$ ). Strikingly, the morphology of $\mathrm{Rlbp}^{+}$Müller glial cell processes was also disrupted in P21 $\mathrm{Plagl1}^{+/ \text {pat }}$ retinas, especially in the rosette structures (Supplementary Fig. ${ }^{3 j}$ ).

To characterize structural deficits in Plagl $^{+/ \text {-pat }}$ Müller glia further, we examined ZO-1 staining, which marks the outer limiting membrane (OLM) of the retina, which is formed by Müller glia end-feet (Fig. 2e). ZO-1 immunostaining showed a clear disruption of OLM adherens junctions in P21 Plagl1 ${ }^{+/ \text {pat }}$ retinas, especially in the vicinity of the rosettes (Fig. 2e). Strikingly, even in the absence of an injury stimulus, Plagl1 ${ }^{+/ \text {pat }}$ retinas had features of an injury response. For instance, around the gaps in the $\mathrm{OLM}, \mathrm{Cd}_{1} 1 \mathrm{~b}^{+}$macrophages and $\mathrm{Iba}^{+}$microglia were observed to infiltrate (Fig. 2f). Finally, retinal vessels labeled with Isolectin-B4 were disorganized, forming abnormal node like structures and tortuous capillaries in P21 and P30 lagll $^{+/ \text {-pat }}$ retinas (Fig. 2g).

The disruption of $\mathrm{Plagll}^{+/ \text {-pat }}$ retinal architecture suggested that retinal activity might also be perturbed, which we assessed by recording full field electroretinograms (ERGs) in P30 juvenile Plagl1 ${ }^{+/ \text {pat }}$ mice, after full visual acuity is reached around P25 ${ }^{37}$ (Fig. 2h-i). Under scotopic (Fig. 
2h) and photopic conditions (Fig. 2i), a and b wave amplitudes were normal in wild-type and Plagl $1^{+/ \text {pat }}$ animals, indicating that the retinal circuitry remained intact in the absence of Plagll function. However, implicit times for both a- and b-waves were prolonged in Plagl1 $^{+/ \text {-pat }}$ mice under scotopic and photopic adaptation (Fig. 2h-i). Altogether, these defects are suggestive of a signal processing dysfunction rather than a global defect in retinal activity.

In summary, Plagl1 ${ }^{+/- \text {pat }}$ retinas have striking morphological disruptions (ectopias, rosettes; Fig. 2j) and display signs of an injury response even in the absence of an insult. Given the prominent expression of Plagll in retinal Müller glia, we focused on how these glial cells are affected by the loss of Plagll for the remainder of this study.

\section{Plagl1 mutant Müller glia undergo insult-independent reactive gliosis}

The morphological defects observed in postnatal Plagl ${ }^{+/ \text {pat }}$ retinas, were strikingly reminiscent of other mutations that result in a loss or dysfunction of mature Müller glia ${ }^{38-40}$, in keeping with the known structural role of these glial cells ${ }^{3,41}$. To determine whether Plagll is required for Müller glia homeostasis, we searched for signs of reactive gliosis, an 'activation' state that normally occurs in response to insult or pathology that is associated with increased expression of intermediate filaments (GFAP, Vimentin) and a decline in Glul activity. In P14 wild-type controls, Vim labeled Müller glia cell bodies, processes, and endfeet, especially those that extend to the GCL (Fig. 3a). Vim immunoreactivity increased in $P l a g l 1^{+/ \text {-pat }}$ retinas, with outer processes and endfeet now clearly labeled, especially in the vicinity of the rosette-like structures (Fig. 3a). Similarly, GFAP expression, which was only expressed in astrocytes lining the GCL in wild-type retinas, was up-regulated in Plagl1 ${ }^{+/ \text {pat }}$ retinas, beginning at $\mathrm{P} 14$, but more strikingly obvious at P21 and P45, especially in the vicinity of the retinal rosettes (Fig. 3b). We also confirmed the reactive state of Plagl1 $^{+ \text {-pat }}$ Müller glia by examining ERK phosphorylation on T202/Y204 (designated pERK), which is normally triggered by growild-typeh factor signaling in reactive Müller glia. pERK protein was detected in Müller glial processes in wild-type retinas, but there was a striking upregulation in expression in cell bodies, including ectopic glia in the ONL, and in glial processes, in P14 Plagl1 ${ }^{+/ \text {pat }}$ retinas, which we confirmed by Western blotting (Fig. 3c,e). Finally, reactive gliosis is also characterized by a reduction of Glul expression, which we also observed in Plagl1 ${ }^{+/ \text {pat }}$ retinas, with gaps in labeled Müller glia in the INL, a reduction that was verified by Western blotting (Fig. 3d,f). 
Touahri et al Plagll sustains Müller glia quiescence

Taken together, these analyses suggest that Plagl $^{+/ \text {pat }}$ Müller glia display several hallmark features of reactive gliosis (Fig. $3 \mathrm{~g}$ ), even in the absence of injury.

\section{Plagl1 mutant Müller glia undergo interkinetic nuclear migration and proliferate ectopically}

Another hallmark feature of Müller glia activation in regenerative species is that glial nuclei undergo interkinetic nuclear migration (INM), moving into the ONL where they then divide to initiate the proliferative phase of the repair process ${ }^{42}$. To assess whether Müller glial undergo INM in Plagl1 $^{+/- \text {pat }}$ retinas, we used Sox9, which marks Müller glia nuclei ${ }^{43}$. While in P21 wildtype retinas, Sox $9^{+}$cells formed essentially a monolayer in the INL, in $\mathrm{Plagl1}^{+/ \text {pat }}$ retinas, Sox ${ }^{+}$ glial nuclei were detected in the INL and in the ONL, especially in the vicinity of the retinal rosettes and ectopia (Fig. 4a,b). Moreover, there was an increase in the total number of Sox $9^{+}$nuclei in Plagl1 ${ }^{+/ \text {pat }}$ retinas, at least in the ONL in the vicinity of the rosettes (Fig. 4b), where an injury-like response has been triggered (see Figs. 2, 3).

Terminally differentiated Müller glia are normally quiescent. Given the increase in Sox $9^{+}$ nuclei in Plagl1 ${ }^{+/ \text {pat }}$ retinas, we questioned whether these glial cells had aberrantly entered the cell cycle. To examine the proliferative response, we analyzed BrdU incorporation into dividing Sphase nuclei in $\mathrm{P} 7$ retinas, when differentiation in the central retina is complete, at P14, when differentiation is complete throughout the retina, and at P21 ${ }^{32}$. BrdU ${ }^{+}$cells were detected at P7 in the central retinas of Plagl1 ${ }^{+/ \text {pat }}$ animals and not their littermate controls, but only in the dysmorphic areas containing clear rosette-like structures (Fig. 4c,d). To identify these proliferating cells, we co-stained with BrdU and Sox9, a Müller glia marker ${ }^{43}$. We found that $95 \%$ of the BrdU cells were Sox $9^{+}$in P7 Plagl1 ${ }^{+/-p a t}$ retinas, suggesting that the majority of dividing cells are glia (Fig. 4c,d). However, because Sox9 is initially expressed in retinal progenitor cells (RPC) and only later becomes Müller glia restricted ${ }^{43}$, to confirm that these dividing cells were not a retained pool of proliferating RPCs, we also co-stained with the RPC marker Pax $6^{44}$. Only $0.4 \%$ of the $\mathrm{BrdU}^{+}$ cells detected in Plagl1 ${ }^{+/ \text {pat }}$ retinas expressed Pax6 in the rosettes (Fig. 4c,d). Thus, the vast majority of proliferating cells in the P7 $\mathrm{Plagl1}^{+/ \text {-pat }}$ retina express Sox9, a Müller glia marker, and not Pax6, an RPC marker.

\section{Plagl1 mutant retinas give rise to new neurons after the normal neurogenic period is over}


In response to injury, Müller glia in all species first undergo reactive gliosis, but only in regenerative species, such as teleost fish and frogs, do these cells re-enter the cell cycle and then undergo neurogenesis ${ }^{7}$. To determine whether proliferating cells in P7 Plagl1 ${ }^{+/ \text {-pat }}$ retinas, which are mainly glia, have also acquired neurogenic competence, we performed a birthdating assay. Proliferating cells were labeled with BrdU at P7, and the fate of these cells was then analyzed at P14 by staining with cell type-specific markers, including Sox9 for Müller glia, Pax6 for amacrine, horizontal and ganglion cells, and Rhodopsin for rods (Fig. 4e). As a thymidine analog, BrdU is incorporated into dividing nuclei in S-phase, and while diluted out of rapidly dividing cells, it permanently labels their non-dividing progeny. Consistent with the $30 \mathrm{~min}$ BrdU assays conducted to assess proliferation (Fig. 4c), we only observed $\mathrm{BrdU}^{+}$cells in $\mathrm{Plagll}^{+/ \text {-pat }}$ and not in wild-type controls in the central retina (Fig. 4e). Strikingly, only $45 \%$ of $\mathrm{BrdU}^{+}$cells stained for the glial marker, Sox9, suggesting that the proliferating cells in the central retina had given rise to other retinal cells (Fig. 4f). Indeed, $23 \%$ of the $\mathrm{BrdU}^{+}$cells were Pax6 $6^{+}$, while $19 \%$ localized to the ONL and expressed Rhodopsin, a rod marker (Fig. 4f).

In aggregate, these data suggest that Plagll is an essential negative regulator of Müller glia activation, including the proliferative response and neurogenic potential of these cells. As Plagll encodes a transcriptional regulator, we next sought to identify downstream transcriptional changes in Plagl1 $^{+/ \text {-pat }}$ retinas that may explain these changes.

\section{Plagl1 regulates the expression of a large network of retinal genes}

To decipher the molecular mechanisms underlying the requirement for Plagll to maintain Müller glia quiescence, we performed bulk RNA-seq on P7 wild-type control and Plagl1 ${ }^{+/ \text {-pat }}$ retinas, at a stage when these glial cells were proliferating ectopically in Plagll $^{+/ \text {pat }}$ retinas. Global transcriptomic analysis identified 2552 differentially expressed genes (DEGs) in Plagl1 ${ }^{+/ \text {-pat }}$ retinas, including up- (1410) and down- (1142) regulated genes, as expected given that Plagll can act as a transcriptional co-/activator or co-/repressor ${ }^{45-48}$ (Fig. 5a). To confirm that P7 Plagl1 ${ }^{+/ \text {-pat }}$ animals were true null mutants, with a silenced maternal allele, we examined Plagll expression levels. Plagll was among the most downregulated genes, but transcripts were only reduced by $50 \%$. However, a survey of read coverage revealed that Plagll exons 5 and 6, covering deleted regions in the mutant allele, were missing in P7 Plagl1 ${ }^{+/ \text {pat }}$ retinas (Fig. 5b), confirming the lack 
of maternal transcription, as previously shown in $P$ lagl1 $1^{+/ \text {pat }}$ mouse embryonic fibroblasts $(\mathrm{MEFs})^{49}$.

Plagll is part of an imprinted gene network (IGN) of biallelically and mono-allelically expressed genes that are tightly co-regulated, the functions of which are poorly understood in the retina in general and in Müller glia in particular ${ }^{31}$. Strikingly, 35/238 IGN genes (15\%) were differentially expressed in Plagl1 ${ }^{+/ \text {pat }}$ retinas (Fig. 5c). Furthermore, a scan of other known imprinted genes (151) identified an additional 6 that were deregulated in Plagl1 ${ }^{+/ \text {-pat }}$ (Ube3a, Casd1, Commd1, Tbcld12, Rian, Peg10) (Fig. 5c), confirming the role of Plagll as a critical regulator of the IGN and highlighting its essential function as a co-regulator of imprinted genes in the retina.

To further classify Plagll-regulated pathways in the retina, we examined gene ontology (GO) term enrichment in the DEG dataset (Supplementary Fig. ${ }^{4 a}$ ). Included amongst the top 30 GO terms in Molecular Function were several terms associated with transcriptional regulation (e.g., "transcription coregulator activity", "DNA-binding transcription factor binding"), while the top 30 Biological Processes enriched GO terms were associated with chromatin modification (e.g. "covalent chromatin modification", "histone modifications"), neuronal differentiation (e.g. "axogenesis", "positive regulation of neuron differentiation"), and neural development ("stem cell maintenance", "maintenance of cell number") (Supplementary Fig. ${ }^{4 a}$ ). Similar GO terms were enriched in Cellular Component analyses, including several terms associated with neuronal differentiation (e.g., "neuron-to-neuron synapse", "post-synaptic density") (Supplementary Fig. 4a). Finally, we also examined Kyoto Encyclopedia of Genes and Genomes (KEGG) pathways, revealing an enrichment of genes associated with neurodegeneration (e.g., "Alzheimer's disease”, “Huntington's disease", "Parkinson's disease”), cancer (e.g. "breast cancer", "prostate cancer", "pancreatic cancer") (Supplementary Fig. ${ }^{4 a}$ ).

In summary, the analysis of Plagl $^{+/ \text {-pat }}$ DEG in the P7 retina suggested that several cellular and molecular processes could have gone awry, including those involved in chromatin and transcriptional regulation, neural development and neuronal differentiation, and neurodegeneration, and cancer. 


\section{Plagl1 regulates a common subset of genes and pathways in different cell types}

Consistent with our finding that Plagll maintains Müller glia quiescence, human PLAGL1 is a tumour suppressor gene, and induces cell cycle exit when misexpressed in cell lines ${ }^{50-53}$ or in the developing nervous system ${ }^{24,54}$. To narrow down genes/pathways potentially responsible for the ectopic Müller glia proliferation observed in P7 Plagl1 ${ }^{+/ \text {pat }}$ retinas, we compared retinal DEGs to those identified in previous Plagl1 ChIP-seq and Plagll loss- and gain-of-function (GOF) RNAseq studies performed in other cell types and tissues (Fig. 5d). Our goal was to identify genes/pathways commonly regulated by Plagll in different cellular contexts. Plagll ChIP-seq using Plagll-overexpressing Neuro-2a cells identified 3859 distinct gene loci bound by Plagl1, in vast excess of the 351 Plagll-regulated genes in these cells, suggesting that Plagl1-binding is not always associated with gene transcription ${ }^{49}$. Nevertheless, we found that 557/3859 (14.4\%) of Plagl1-bound gene loci in Neuro2a cells were amongst the DEGs in P7 Plagl1 ${ }^{+/ \text {pat }}$ retinas, while 34/351 (9.7\%) of the genes induced by Plagll overexpression in Neuro2a cells were also deregulated in Plagl1 $1^{+/ \text {pat }}$ retinas (Fig. 5d). An even larger proportion of DEGs in P7 Plagl1 ${ }^{+/ \text {-pat }}$ retinas were shared with those that were deregulated when Plagll was overexpressed in embryonic cortical progenitors $(232 / 1400 \text { genes }=16.6 \%)^{55}$ (Fig. 5d). Finally, a comparison to RNA-seq data of $\mathrm{Plagl1}^{+/ \text {-pat }}$ mouse embryonic fibroblasts (MEFs) 49 identified 36/238 genes (15.1\%) as commonly deregulated in the P7 Plagl1 ${ }^{+/ \text {pat }}$ retina (Fig. 5d).

We next assessed GO term and KEGG pathway enrichment in the commonly deregulated genes in each of these datasets (Fig. 5d; Supplementary Fig. ${ }^{4 b}$ ). Of the GO biological process terms, proliferation was a top de-regulated term amongst retinal DEGs and the Plagll ChIP-seq data, while several neuronal differentiation terms were also shared with Plagll $^{+/ \text {pat }}$ retinas. One neuronal differentiation GO term was also significantly enriched amongst the DEG in Plagl1 $^{+/ \text {-pat }}$ mutant retinas, Plagl1 ChIP-seq data and Plagll GOF in cortex. Finally, KEGG pathway analysis identified Notch signaling and various cancer terms as the only shared pathways between $\mathrm{Plagl1}^{+/}$ ${ }^{\text {pat }}$ mutant retinas, Plagl1 ChIP-seq and Plagll GOF (padj<0.05).

Taken together, these comparative studies suggest that Plagll controls common gene sets across cell types, which could be involved in retinal homeostasis and tissue morphogenesis, as well as proliferation and neuronal differentiation, processes that we investigated further below in Plagl1 ${ }^{+/ \text {pat }}$ retinas. 


\section{Plagl1 globally reduces chromatin accessibility without altering the expression of critical glial genes}

"Covalent chromatin modification" and "histone modification" were the two top GO biological processes associated with DEGs in P7 Plagl1 ${ }^{+/ \text {pat }}$ retinas (Supplementary Fig. ${ }^{4 a}$ ). To test whether the transcriptomic changes observed in $\mathrm{Plagll}^{+/ \text {pat }}$ are due to epigenetic regulation, we used ATAC-seq to identify nucleosome-sparse (open) genomic DNA ${ }^{56}$. ATAC-seq was performed on $\mathrm{P} 7$ control and Plagl1 ${ }^{+/ \text {pat }}$ retinas, which identified 833 differentially accessible regions (DARs). Strikingly, the vast majority of the Plagl1 ${ }^{+/ \text {pat }}$ DARs (814/833) displayed decreased accessibility in Plagl $1^{+/ \text {pat }}$ retinas, while 19 had increased accessibility (Fig. 5e). We compared DARs and DEGs in Plagl1 ${ }^{+/ \text {pat }}$ retinas by combining ATACseq and RNAseq data. We limited the DARs list to 311 peaks located within $5000 \mathrm{bp}$ from the nearest transcription start site (TSS) and compared to the RNAseq data for the associated genes. As expected, the majority of DARs (308/311) were associated with the decrease of accessibility in Plagll $^{+/ \text {pat }}$. While no increased DARs (0/3) overlapped with either up- or down-regulated genes, out of 308 decreased accessibility DARs, 15 overlapped with down-regulated genes and 36 with up-regulated genes in Plagl1 ${ }^{+/ \text {pat }}$ (Fig. 5f). Interestingly, several of the upregulated genes were identified as genes involved in various aspects of Müller glia homeostatic functions and/or the injury response (Fig. 5f).

Taken together, these comparative studies suggest that Plagll controls, at the transcriptional level, gene sets involved in retinal homeostasis, which we investigated further below in Plagl1 $^{+/ \text {pat }}$ retinas.

\section{Additional signs of a retinal injury occur in $\mathrm{Plagl1}^{+/-p a t}$ retinas}

Müller cell gliosis occurs in response to retinal injury or disease, and in chronic conditions, contributes to a neurodegenerative process ${ }^{57}$. Activation of Müller glia in Plagl1 $^{+/ \text {-pat }}$ mutants suggested that there may be additional shared features with other retinal degeneration models. We thus compared the $\mathrm{Plagll}^{+/ \text {pat }}$ transcriptome to neurodegenerative models (Supplementary Fig. ${ }^{4 c-}$ $\left.{ }^{e}\right)$. We first examined Norrin $(N d p)$ knock-outs (KOs), which are a model of retinal hypovascularization and chronic hypoxia due to a disruption of the blood-retinal-barrier (BRB), and which also display reactive gliosis ${ }^{58}$. Of the 92 Müller glia enriched genes in $N d p$ KOs, 19 (20.7\%) genes were among the DEGs in P7 Plagl1 ${ }^{+/ \text {pat }}$ retinas, including Vegf, a critical regulator of 
angiogenesis. In $r d 10$ mutants, which undergo a progressive loss of rod photoreceptors, of the 20 Müller glia-enriched genes, $4(20 \%)$ were also deregulated in P7 Plagl1 ${ }^{+/ \text {pat }}$ retinas (Supplementary Fig. ${ }^{4 d}$ ). Included was Vim, a marker of reactive gliosis, as well as Sox9, a glialspecific retinal gene that is upregulated in injury ${ }^{8}$. Finally, in $R d s / \operatorname{Prph} 2$ mutant mice, treatment with CNTF induced GFAP expression and reactive gliosis, and transcriptomic analyses identified 4 Müller glia-enriched genes ${ }^{59}$, including $V_{s x} 2$, a retinal progenitor cell (RPC) gene upregulated in injury, which was amongst the in P7 Plagl1 ${ }^{+/ \text {-pat }}$ retinal DEGs (Supplementary Fig. ${ }^{5 \mathrm{e}}$ ).

The transcriptomic resemblance of $\mathrm{Plagl1}^{+/ \text {-pat }}$ retinas to neuronal degeneration models and injury prompted a more detailed comparison to the recent comprehensive analysis of the Müller glia response to injury ${ }^{7}$.

\section{Plagl1 controls a subset of Müller glia specific genes}

The Müller glia proliferation phenotype in addition to the expression profile suggest a prominent role of Plagll in maintaining Müller glia in quiescence. To better understand Plagll function, we analysed Plagl1 ${ }^{+/ \text {pat }}$ DEGs that were expressed in Müller glia sorted from GLASTCreERT2;Sun1-sGFP mice ${ }^{7}$. In this large dataset, of 2263 genes expressed in Müller glia, 372 were among the P7 Plagl1 ${ }^{+/ \text {pat }}$ retinal DEGs (Fig. 5g). Among these 372 DEGs, 275 were upregulated while 97 were down-regulated (Fig. 5g). Included were glial determinants of the Nfi $(\text { Nfib, Nfix })^{60}$ and Sox (Sox2, Sox4, Sox6 Sox8, Sox9) ${ }^{61}$ families, the hippo pathway effectors Tead1 and Tead2, Notch pathway genes (Notch1, Notch2, Hes1, Hes5, Snbo2), other signalling pathways (Sema5b, Rgs2, Fgfr1, Fzd5), ribosomal genes involved in translation (Rps19, Rps5, Rps4x, Rpl23, Rpl14, Rpl7l1, Rpl35, Rpl29) and metabolism/catabolism related genes (Trhde, Car2, Ubc, Ctsh) (Fig. 5h).

To further characterize the state of Plagl1 ${ }^{+/ \text {pat }}$ Müller glia, we compared DEGs to the transcriptomes of NMDA and LD injured retinas. Comparing DEGs in the 3 conditions showed that of the 2552 genes differentially expressed in $P$ lagl $1^{+/ \text {-pat }}$ retinas, 637 are injury responding genes that are also DEGs in both NMDA and LD treatments (Fig. 6a). Next, we compared the behaviour of these differentially expressed genes in Plagl1 $^{+/ \text {pat }}$ and injured Müller glia at different time points. We identified: (1) genes that were altered in the same direction in Plagll $^{+/ \text {-pat }}$ and injured Müller glia (up-or down-regulated), (2) genes that were differentially expressed in injury and not in Plagll $^{+/ \text {-pat }}$ retinas (or vice versa), and (3) genes that were up-regulated in Plagl1 ${ }^{+/ \text {-pat }}$ 
retinas and down-regulated in injury (or vice versa) (Fig. 6b, Supplementary Fig. ${ }^{5 a}$ ). Interestingly, we found that several Notch pathway genes were upregulated in P7 Plagl1 ${ }^{+/ \text {pat }}$ mutant retinas (Fig. 6c, Supplementary Fig. ${ }^{5 b, c}$ ) whereas they were downregulated in NMDA and LD injured Müller glia (Fig. 6d). Included were Notch downstream effectors, Hes 1 and Hes5, the receptors Notch1 and Notch2, and the ligand Dll1, while Serr and Jag2 ligands were only up-regulated in Plagl1 ${ }^{+/-}$ pat (Fig. 6c,d, Supplementary Fig. ${ }^{5 b, c}$ ).

To confirm that an increase in Notch signaling did occur in Plagl1 ${ }^{+/ \text {pat }}$ Müller glia, we performed RNAscope on P7 control and Plagl1 ${ }^{+/ \text {pat }}$ retinas. We hybridized labeled riboprobes to retinal sections to detect transcripts for Hes1, Hes5 and the Müller glia marker Sox9. As expected, both Hes5 and Hes 1 were expressed in the INL, co-localized with Sox9, and strikingly, their expression levels were increased in P7 Plagl1 ${ }^{+/ \text {pat }}$ Müller glia (Fig. 6e). In addition, this upregulation was confirmed by RT-qPCR confirmed in P7 Plagl1 ${ }^{+/ \text {pat }}$ mutant retinas (Supplementary Fig. ${ }^{5 d}$ ). These data indicate that Plagll loss of function results in Notch signalling pathway increase in P7 Müller glia.

\section{Upregulation of cell cycle genes in $\mathrm{Plagl1}^{+/-p a t}$ retinas}

In contrast to injured murine Müller glia that respond with limited or no proliferation, Plagl1 ${ }^{+/ \text {pat }}$ Müller glia undergo robust proliferation at P7 (Fig. 4). We thus assessed the potential deregulation of cell cycle genes in Plagl1 $1^{+/ \text {pat }}$ retinas by compiling a list of 616 genes involved in cell cycle regulation, including all genes in the GO category "GO0007049: cell cycle" and a functional classification of additional cell cycle genes based on GO categories and KEGG pathways using DAVID. Enriched categories were organized into clusters based on semantic similarity. Of the 616 compiled genes, 90 genes were deregulated $(14.6 \%)$ in Plagl1 $^{+/ \text {pat }}$ retinas, including both down-regulated (50 genes) and upregulated (40 genes) genes, as depicted in a heatmap (Fig. 6f). Among the downregulated genes were known negative regulators of the cell cycle $(C d k n 2 d)$, while positive regulators were amongst the upregulated genes $(C d k 6, C d k 11 b$, Pard3, Pak4) (Fig. 6f).

To identify potential regulators of Müller glia proliferation, we screened the 90 DEGs involved in cell cycle regulation for Müller glia specific genes. We identified 11 cell cycle genes specifically expressed in Müller glia and differentially expressed in $P l a g l 1^{+/ \text {-pat }}$. Amongst these genes Rgs2, Arf6, Klh113 were down-regulated while Cdk6, Src, Rcc2, Mad2l2, Pdcd6ip, 
Trp53bp2 and Pak4 were up-regulated in P7 Plagl1 ${ }^{+/ \text {pat }}$ (Fig. 6g). 64 genes were expressed in Müller glia but not specific to, with 27 genes upregulated and 37 down regulated in Plagl1 $^{+/ \text {-pat }}$ (Table 1). Finally, we analyzed the expression of these genes in NMDA/LD injured Müller glia and compared their transcriptional behaviour following injury to their expression in Plagll $^{+/- \text {pat }}$. Interestingly, Pak4 was up-regulated in $P l a g l 1^{+/ \text {pat }}$ and not differentially expressed in NMDA/LD injured Müller glia. In contrast, Numa1, Cep131 were up-regulated in Plagl1 ${ }^{+/ \text {pat }}$ and downregulated in NMDA/LD injured Müller glia, while Rps3, Rab11a, Zwint, Uba3 and Lig4 were down-regulated in Plagl1 ${ }^{+/ \text {pat }}$ and up-regulated in NMDA/LD injured Müller glia (Supplementary Fig. $\left.{ }^{6 a-b}\right)$.

Taken together these data support the notion that the loss of Plagll function results in a robust proliferation of Müller glia at P7 in the retina. Furthermore, our transcriptional analysis suggests Plagll acts upstream genes and signalling pathways that control Müller glia proliferation especially Notch signalling pathway genes, as well as $L h x 2$, and Yap genes Tead1,2 and a network of cell cycle genes.

\section{DISCUSSION}

In the retina, Plagll is expressed in retinal progenitor cells, where it plays a role as a negative regulator of cell number control, ensuring that appropriate numbers of rod photoreceptors and amacrine cells are generated ${ }^{24}$. In addition, Plagll is expressed in subsets of mature retinal cells, including adult Müller glia ${ }^{62}$, where its functions have not been studied. Here we found that Plagll is expressed in a dynamic fashion in Müller glia post-injury, with a rapid and transient upregulation that is followed by a more long-term decline. To study Plagll function, we examined Plagl1 ${ }^{+/ \text {-pat }}$ animals, which we confirmed to be null mutants, revealing that they develop defects in retinal architecture and visual function beginning at perinatal stages, which correlate with a reactive gliotic phenotype of Müller glia. Furthermore, we found that Plagl1 ${ }^{+/ \text {pat }}$ Müller glia proliferate in an injury-independent fashion, and give rise to new inner retinal neurons and photoreceptors postinjury. Based on transcriptomic analyses, we found that Plagll is a negative regulator of several pro-proliferative and pro-glial pathways in the retina, including Notch signaling. The loss of Plagll thus initiates the three critical steps in regeneration in murine Müller glia, which include reactive gliosis, re-entry into the cell cycle, and neurogenesis. 
In addition to the shared structural abnormalities between Plagl1 ${ }^{+/- \text {pat }}$ and other retinal degenerative models, we observed several overlapping transcriptomic changes with $N d p, r d 10$, and $r d s$ mutants, as well as some overlap with the NMDA injury model. These findings are in keeping with the central role that Müller glia dysfunction plays in retinal degeneration, including in human disease, including in macular telangiectasia type 2 (MacTel-2) ${ }^{63}$. We attribute the Plagl1 ${ }^{+/ \text {pat }}$ degeneration-like phenotype to a requirement for Plagll in Müller glia because the overlapping phenotypes observed after Müller glia are ablated, including retinal rosettes, vascular defects, and abnormal ERG recordings ${ }^{38}$.

The role of Plagll in sustaining quiescence is consistent with its role as a tumor suppressor gene. In humans, PLAGL1 localizes to a chromosomal region (6q24-25) linked to growild-typeh inhibition, its ability to induce cell cycle arrest and apoptosis when misexpressed in epithelial cell lines ${ }^{51,53}$, and its inactivation in breast ${ }^{53}$, pituitary ${ }^{64}$, head and neck ${ }^{65}$, and ovarian carcinomas 66,67. However, our study provides the first evidence of an in vivo role for Plagll in maintaining non-malignant somatic cells in quiescence. Plagll null mutant retinas also resemble several other Müller glia-specific conditional KOs (cKOs), such as Dicer cKOs, which also have retinal dysmorphology and defects in retinal function, although hey do not display ectopic proliferation 68 . Transcription factors of the Nfi family can also promote a glial identity and cell cycle exit when misexpressed in late RPCs ${ }^{60}$. Interestingly, misexpression of murine Plagll in Xenopus ${ }^{23}$ and mouse ${ }^{24}$ also promotes a Müller glial cell fate, but only in mouse is PLagl1 necessary and sufficient to promote cell cycle exit. Taken together with this study, we conclude that Plagll plays a role not only in the development of Müller glia, but also in maintaining the quiescence of these cells at postnatal stages.

However, ectopic proliferation of Plagl1 ${ }^{+/ \text {pat }}$ Müller glia was transient, only observed at P7 and not later, and even at P7, not all Müller glia had re-entered the cell cycle, even though most of these cells express Plagll. This finding is similar to the transient proliferative response of mammalian Müller glia to injury ${ }^{69-71}$, and suggests that there may be distinct populations of Müller glia that respond to injury. In addition, there is growing support that there are active inhibitory processes that have to be overcome for Müller glia to re-enter the cell cycle. One example is $C d k n 1 c$, which plays a critical role in preventing cell cycle re-entry of Müller glia. However, $C d k n 1 c$ was not downregulated in Plagll mutant retinas, although $C d k n 2 d$, another cell cycle 
inhibitor, was reduced in expression, and multiple positive cell cycle regulators were also elevated (Fig. 6f).

Another conundrum is the increased expression of $N f i b / c / x$ in Plagll mutant retinas, which promote a glial identity in the retina ${ }^{60}$, but are also required to promote quiescence, although injury response can induce ectopic proliferation in these mutants ${ }^{7}$. In contrast, in this study, we showed that Müller glia proliferate ectopically in $P l a g l 1^{+/ \text {-pat }}$ retinas even without injury. Similarly, Lhx2 is required to prevent reactive gliosis in an injury-independent fashion ${ }^{72}$, but we found that $\operatorname{Lh} x 2$ is upregulated in Plagll mutants. One possibility is that Plagll acts downstream of $N f i$ and Lhx2 transcription factors, such that its loss has a dominant action in maintaining Müller glia quiescence.

Plagll is a maternally imprinted gene that is expressed in a parent-of-origin-specific fashion (i.e., paternal allele only) due to DNA methylation of the maternal allele, a modification that first appears in the germ cells ${ }^{73}$. Imprinting affects $<1 \%$ of all genes in placental animals, including mammals ${ }^{74,75}$. Imprinted genes are heterogeneous in function (e.g., transcription factors, signaling molecules, cell cycle regulators), but recent studies suggest they may share a common function in the maintenance of cellular quiescence ${ }^{27}$. A meta-analyses of 85 known IGs, revealed that these genes are co-regulated; Plagll is part of an IGN of 13 imprinted genes $(\mathrm{Meg} 3, \mathrm{Ndn}$, Grb10, Dlk1, Igf2, Cdkn1c, Zac1, Peg3, Mest, Nnat, Asb4, H19, Ppp1r9a) that are tightly coregulated $^{27,31}$. Strikingly, many of these IGN genes are negative regulators of cell growild-typeh (Igf2 a notable exception) whether the imprint is maternal or paternal ${ }^{27,31}$. Of these, $D l k 1^{29}$ and $C d k n 1 c^{30}$ maintain adult neural stem cell (NSC) quiescence in the forebrain. Notably, two IGN genes become biallelically expressed in adult somatic cells; Dlk1 in adult $\mathrm{NSCs}^{29}$; Igf2 in meninges, choroid plexus and vasculature ${ }^{76}$. In contrast, our analysis of transcript reads revealed that Plagll remains mono-allelically expressed in the postnatal retina.

Expression of several of these IGN genes is also associated with cell cycle withdrawal/differentiation in vitro ${ }^{27}$. However, our work demonstrating that Plagll maintains retinal Müller glia in quiescence is the first to establish this relationship in vivo. Notably, Plagll mutant MEFs do not proliferate ectopically ${ }^{49}$, suggesting that Plagll sustains quiescence only in a subset of somatic cells in vivo, and one intriguing possibility is that this function is restricted to those cells with stem cell potential. Consistent with this idea, two members of the IGN, Dlk $1^{29}$ and $C d k n 1 c^{30}$, maintain adult neural stem cell quiescence in the forebrain. Interestingly, of the imprinted genes in the Plagll IGN, only Dlkl was differentially expressed in $P l a g l 1^{+/ \text {pat }}$ retinas, 
with an increase in transcript levels. Dlk1 is a negative regulator of Notch signaling ${ }^{77}$, but as multiple downstream effectors of Notch are increased in $\mathrm{Plagl1}^{+/ \text {-pat }}$ retinas, it does not appear that Dlk1 has a functional role in inhibiting Notch signaling in these animals. Notably, Dlk1 (also known as Pref1) can activate ERK signaling in adipocytes ${ }^{78}$, and as ERK signaling is upregulated in Plagll ${ }^{+/ \text {pat }}$ Müller glia, Dlkl may contribute to this process. ERK signaling is activated downstream of receptor tyrosine kinases (RTKs) in response to growth factor signaling (e.g., EGF, IGF, FGF2), and activation of this pathway is a universal feature of reactive gliosis in all species 6 .

In conclusion, we have identified Plagll as a critical regulator of mammalian Müller glia quiescence, one of the few genes that when mutated initiates a proliferative glial response. There are clear implications for regenerative medicine, in which the goal is to initiate the endogenous Müller glial repair response that occurs in fish and frogs.

\section{METHODS}

Animals. Animal procedures were approved by the University of Calgary Animal Care Committee (AC11-0053) and later by the Sunnybrook Research Institute (16-606) in compliance with the Guidelines of the Canadian Council of Animal Care and conformed to the ARVO statement for the Use of Animals in Ophthalmic and Vision Research. To stage embryos, we considered the morning of the vaginal plug as embryonic day (E) 0.5 . Plagl1 ${ }^{+/ \text {pat }}$ animals were generated by crossing Plagl1 ${ }^{+/ \text {mat }}$ males (obtained from Laurent Journot ${ }^{31}$ ) with C57/B16 wild-type females (\#000664, Jackson Laboratory, ME, USA). Genotyping was performed with the following primers: Plagll mutant allele: forward (5'-ATG GCT TCT GAG GCG GAA AG-3') and reverse (5'-AAA GGC TCC AAA GGC TCC AAG G-3'); Plagll wild-type allele: forward (5'-TCG TCA CAC CAA GAA GAC CCA C-3') and reverse (5'-AAA GGC TCC AAA GGC TCC AAG G-3'); CD1 mice (Charles River Laboratories, Senneville, QC, Canada) were used for explant experiments.

Electroretinogram (ERG). Animals were dark-adapted for one hour prior to ERG recordings. ERGs were recorded under dim red light. Stimulation and acquisition were performed with an Espion $E^{2}$ system (Diagnosys LLC) (flash duration $10 \mu \mathrm{s}$, bandpass filtering $0.3 \mathrm{~Hz}-3 \mathrm{Khz}$ ), as described ${ }^{79,80}$. Briefly, scotopic intensity responses were measured using increasing light flash 
Touahri et al

Plagll sustains Müller glia quiescence

pulses of increasing strength (-5.22 to $\left.2.86 \log \mathrm{cds} / \mathrm{m}^{2}\right)$ and photopic intensity responses $\left(30 \mathrm{~cd} / \mathrm{m}^{2}\right.$ background light) used increasing flash strengths from -1.6 to $2.9 \log \mathrm{cds} / \mathrm{m}^{2}$.

Optical coherence tomography (OCT). Mice were anesthetized with 2\% isoflurane general anaesthesia after their pupils were dilated using tropicaminde topical drops (Mydriacyl 1\%; Alcon Canada Inc). Eyes were imaged using the Spectralis optical coherence tomography (OCT) and confocal scanning laser ophthalmoloscopy (cSLO) system mounted with an additional 25-diopter lens (Heidelberg Engineering, Germany). Normal saline was applied to the mice corneas every 2 minutes to keep it hydrated. OCT was performed on a $30^{\circ} \times 20^{\circ}$ area of $\mathrm{P} 21$ mouse retinas. A total of 31 scans were acquired with an average of 20 frames per scan. Infrared cSLO scans (IR-cSLO) were acquired $(\lambda=815 \mathrm{~nm})$ with an average of 30 frames per scan to assess the retinal fundus of Zac1 mutant and controls. Retinal scans were exported from the HEYEX software (Heidelberg Engineering, Germany) as tiff images and processed using ImageJ software (NIH, USA).

Tissue processing. Retinal tissues were drop fixed in 4\% paraformaldehyde (PFA)/1X phosphate buffered saline (PBS) at $4{ }^{\circ} \mathrm{C}$ overnight for dissected retinas or eyes or 3 hours for retinal explants. Fixed tissues were then rinsed 3 x 10 min in 1 X PBS and cryopreserved in $20 \%$ sucrose/1X PBS overnight at $4^{\circ} \mathrm{C}$. For cryosectioning, tissues were embedded in O.C.T TM (Tissue-Tek ${ }^{\mathrm{T}}$, Sakura Finetek U.S.A. Inc., Torrance, CA). Cryosections were cut at 10-12 microns on a Leica CM3050s cryostat (Leica Biosystems, Buffalo Grove, IL, USA) and collected on Fisherbrand ${ }^{\mathrm{TM}}$ Superfrost ${ }^{\mathrm{TM}}$ Plus slides (Thermo Fisher Scientific, Markham, ON). Collected slides were either processed directly or stored at $-20^{\circ} \mathrm{C}$ in a tape-sealed slide box.

Immunohistochemistry. Cryosections were blocked for $1 \mathrm{hr}$ at room temperature in blocking solution: 10\% normal goat serum in PBST (1X PBS/0.1\% Triton X-100). Primary antibodies were diluted in blocking solution and incubated at $4^{\circ} \mathrm{C}$ overnight. The following primary antibodies were used: rabbit anti-Arr3 (1/500, Millipore \#AB15282), rat anti-BrdU (1/500, Serotec \#OBT0030S), mouse anti-Brn3a (1/500, Chemicon \#5945), mouse anti-calbindin (1/1000, Sigma \#9848), rabbit anti-calretinin (1/2000, Swant \#76699/4), rat anti-CD11b (M1/70, 1/500, Abcam \#ab8878), mouse anti-Rlbp1 (1/500, Abcam \#15051), rabbit anti-GFAP (Sigma \#G9269), rabbit anti-Glul (1/500, Abcam \#73593), rabbit anti-Iba1 (1/500, Cat\#019-19741), rabbit anti-M-opsin 
Touahri et al

(1/250, Millipore \#5405), rabbit anti-Pax6 (1/500, Convance \#PRB-278P), Rabbit anti-pERK (Map kinase p44/42 phospho-Thr202/Tyr204) (1/500, Cell signaling \#4370), mouse antirhodopsin (1/500, Chemicon \#MAB5356), rabbit anti-S-opsin (1/250, Millipore \#5407), rabbit anti-Sox9 (1/500, Millipore \#AB5535), mouse anti-syntaxin (1/500, Sigma \#S 0664), mouse antivimentin (1/500, Sigma \#V5255), or anti-ZO-1 (1/100, ThermoFisher \#33-9100), Slides were washed $3 \times 10 \mathrm{~min}$ in PBST and then incubated in secondary antibodies conjugated to Alexa-568, Alexa-488 or Alexa-647 diluted at 1/500 in PBST for $1 \mathrm{hr}$ at room-temperature, followed by $3 \mathrm{x}$ 10 min washes in PBST.

Isolectin staining. Eyes were dissected and retinas were flatmounted or processed for sectioning as described. Wholemount retinas were fixed in 4\% PFA/1X PBS for 2 hours. Flatmounted retinas or cyrosections were fixed in ice cold $70 \%$ ethanol for $30 \mathrm{~min}$. Fixed tissue was then incubated in Isolectin B4 (1/250, Sigma \#L2140) in PBST overnight at $4^{\circ} \mathrm{C}$, washed $3 \mathrm{X} 10 \mathrm{~min}$ in PBST. Wholemount retinas were mounted on microscope slides and mounted tissues or sections were coverslipped in Aquapolymount.

BrdU labeling. To label S-phase cells, animals were injected intraperitoneally with $100 \mathrm{~g} / \mathrm{g}$ body weight BrdU (Sigma, Oakville ON) either 30 min before sacrifice for proliferation assays or at P7 for birthdating assay. Tissues were processed for anti-BrdU staining as described above except for the addition of a pre-treatment step with $2 \mathrm{~N} \mathrm{HCl}$ for 15 min at $37^{\circ} \mathrm{C}$ prior to blocking.

Retinal explants. Retinas were dissected and the RPE removed from P0 eyes in ice-cold PBS. Retinas were flattened on $0.25 \mu \mathrm{m}$ Nucleopore membranes (Whatman \# 110409) in 6 well plates and cultured for 14 days in vitro (DIV) at $37^{\circ} \mathrm{C}, 5 \% \mathrm{CO}_{2}$ in retinal explant medium. (50\% Dulbecco’s Modified Eagle Medium (DMEM, Wisent; \#319-005-CL), 25\% Hanks’ Balanced Salt Solution (HBSS, Gibco; \#24020-117), 25\% heat inactivated horse serum (ref), $200 \mu \mathrm{M}$ LGlutamine (Wisent; \#609-065-EL), 0.6 mM HEPES (Wisent; \# 330-050-EL), 1\% Pen/Strep (Wisent; \# 450-201-EL). For lineage tracing, 4-hydroxytamoxifen (4-OHT) (Sigma-Aldrich; \#H6278) was added to the retinal explant media after $7 \mathrm{DIV}$ at $5 \mathrm{mM}$ final concentration. Half of the media was removed and replaced with fresh media every day with a $2 \mathrm{X}$ concentration of 4OHT. Explants were harvested after 14 DIV. 
RNA extraction. Total RNA was extracted from dissected retinas using Trizol® RNA Isolation Reagent (Thermo Fisher Scientific; \#15596-026), following the manufacturer's instructions except the overnight incubation step was reduced to $30 \mathrm{~min}$ at $-80^{\circ} \mathrm{C}$. RNA quality was assessed using an Agilent 2100 Bioanalyzer (Agilent Technologies, Palo Alto, CA, USA).

RNA sequencing. For RNA-Seq $8 \mathrm{Plagll}^{+/ \text {-pat }}$ and 4 wild-type littermates were analyzed. $500 \mathrm{ng}$ RNA per sample was sequenced on Illumina NextSeq500 Platform. 75 base single-end sequence reads were generated on NEBNext Ultra II Directional RNA Library Prep Kit for Illumina. Basecalling and demultiplexing was done using IIllumina CASAVA 1.9 pipeline. Reads were mapped to mouse genome (Ensembl, GRCm38) using hisat2 version 2.1 .0 with a mapping rate close or over $98 \%$ in each sample.

Differential expression and clustering analysis. Differentially expressed genes were detected using DESeq2 1.24.0 Bioconductor package https://bioconductor.org/packages/release/bioc/html/ DESeq2.html (Love et al., 2014, p. 2) as described in package vignette. Genes with adjusted pvalues (Benjamini-Hochberg adjustment for multiple comparisons) (Benjamini and Hochberg, 1995 ) less then 0.05 (5\% chance of gene being a false positive) and over 1.5 fold change in either direction were selected as differentially expressed.

Gene Ontology analysis. Over-represented gene ontology (GO) terms and Kyoto Encyclopedia of Genes and Genomes (KEGG) (Kanehisa and Goto, 2000) pathways were detected using clusterProfiler 3.12.0 Bioconductor package (Yu et al., 2012). The analysis was done separately was on the lists of up- and down-regulated genes.

ATAC-seq. ATAC-seq was performed using 75,000 cells as input material for the library preparation with Active motif kit (Catalog No. 53150) following the manufacturer's instructions. 75 bp paired-end sequencing was performed on Illumina NEXTSEQ500. Reads were mapped to the reference genome (Ensembl, GRCm38) using bowild-typeie2 short read aligner version 2.3.5.1. Areas of open chromatin were predicted using Macs2 v.2.2.7.1 based on alignment files filtered of mitochondrial reads. Differential enrichment was detected using DiffBind 
Bioconductor package. Data was normalized using Trimmed Mean of Medians (TMM) normalization native to EdgeR Bioconductor package. In total, differential binding tests were applied to 56771 peaks. The peak had to be present in at least 2 samples within experimental group to be included into the analysis. False discovery rate (FDR) below 0.05 was a threshold to consider the peak differentially bound. No peaks were below 0.05 FDR threshold. DiffBind results were annotated with nearest promoter, gene symbol, entrez_id and gene description using ChIPpeakAnno Bioconductor package. Term enrichment analysis was conducted using 2 different approaches: 1) over-representation analysis (ORA) conducted using geneSCF software and 2) Gene set enrichment analysis (GSEA). Genes with TSS located within 5000 bp of DARs were used in ORA. The input for GSEA was the list of all genes within $5000 \mathrm{bp}$ from the nearest TSS sorted by the Fold Change. The GSEA enrichment analysis was conducted using ClusterProfiler v.3.18.1 Bioconductor package.

ATAC-seq and Zac1 RNA-seq integration. ATAC-seq and RNA-seq were integrated by overlapping the lists of significantly changed genes and DARs. Genes or DARS with adjusted pvalues below 0.05 were considered significant. The list of DARs was limited to 311 peaks located within $5000 \mathrm{bp}$ from the nearest TSS.

RT2 Real-time Quantitative Reverse Transcription-PCR. For complimentary DNA (cDNA) synthesis, $0.5 \mu \mathrm{g}$ of total RNA was converted using an $\mathrm{RT}^{2}$ first strand kit (Qiagen, \#330401) following the manufacturer's instructions. qPCR reactions were performed using a CFX384 cycler (Biorad Laboratories, Canada) using a RT² SYBR® Green PCR Master Mix (Qiagen; \#330500) following the manufacturer's instructions. The following $\mathrm{RT}^{2}$ qPCR primers were used: Sox 9 (PPM05134D), Lhx2 (PPM31533A), Hes1 (PPM5647A), Hes5 (PPM31391A) and three reference genes for normalization: Gapdh (PPM02946E), B2m (PPM03562A), and Hrpt (PPM03559F). The $\Delta \Delta \mathrm{Ct}$ method was used to determine relative gene using the Biorad CFX manager software.

RNAscope. Double fluorescent in situ hybridization were performed using a RNAscope ${ }^{\circledR}$ Multiplex Fluorescent Detection Kit v2 (ACD; \#323110) according to the manufacturer's directions. ACD probes used included: Mm-Sox9 (C2: 401051-C2), Mm-Hes1 (\#417701), MmHes5 (400991 -C2), and Mm-Plagl1 (C1: 462941). Opal ${ }^{\mathrm{TM}} 570$ (Akoya; \#FP1488001KT; 1:1500) 
was applied for channel 1 and Opal ${ }^{\mathrm{TM}} 520$ (Akoya; \#FP1487001KT; 1:1500) was applied for channel 2. Retinal sections were counterstained with DAPI and mounted in Aquapolymount as described.

Western blotting. Retinas and retinal explants were collected from adult and postnatal pups at the indicated stages, lysed in RIPA buffer with protease (1x protease inhibitor complete, $1 \mathrm{mM}$ PMSF) and phosphatase (50 mM NaF, $1 \mathrm{mM} \mathrm{NaOV}$ ) inhibitors, and $10 \mu \mathrm{g}$ of lysate was run on $10 \%$ SDSPAGE gels for Western blot analysis as described previously (Ma et al., 2007). Primary antibodies included Gfap (Sigma, \#G9269, 1/10.000), Vimentin (Sigma, \#V5255, 1/10.000), pERK, Glutamin Synthetase (Abcam, \#ab73593, 1/10.000) and Actin (Abcam; \#ab8227, 1/10.000). Densitometries were calculated using ImageJ. The average values of normalized expression levels were plotted.

Image acquisition and processing. Images were captured on a Leica DMRXA2 optical microscope (Leica Microsystems Canada Inc., Richmond Hill, Ontario, Canada) using LasX software. Confocal images were acquired using a Leica SP8 spectral confocal microscope (Leica Microsystems CMS GmbH). The images were prepared using Adobe Photoshop CC 2017 (Adobe Systems Inc., San Jose, CA, USA). For Plagl1 ${ }^{+/ \text {pat }}$ retinal transverse sections, all areas and sections containing rosettes and/or ectopia were imaged, the equivalent areas/ sections number were imaged for the wild-type littermate and used as controls.

Statistical analysis. Cell counts were performed on a minimum of three retinas for each genotype, and a minimum of three sections from each eye. The $n$ numbers and statistical tests used for each count were indicated in the figure legends. Graphs and statistics were generated using GraphPad Prism Software 8 (GraphPad Inc., La Jolla, CA). All data expressed as mean value \pm standard error of the mean (SEM). In all experiments, a $\mathrm{p}$ value $<0.05, * * \mathrm{p}<0.01, * * * \mathrm{p}<0.005$, and $* * * * \mathrm{p}<$ 0.001 .

\section{ACKNOWLEDGEMENTS}

CS holds the Dixon Family Chair in Ophthalmology Research at Sunnybrook Research Institute. This project is currently supported by funding to CS from Natural Sciences and Engineering Research 
Touahri et al Plagll sustains Müller glia quiescence

Council of Canada Discovery Grant-RGPIN-2017-03649 and Canadian Institutes of Health Research (CIHR) Operating Grant MOP-142338. YT was supported by a U of Calgary Eyes High Fellowship, MH by an Alberta Innovates Health Solution (AIHS) summer studentship, LA and NT by a CIHR/Alberta Children's Hospital Research Institute training grant, and RD by a CIHR Canada Hope Fellowship.

\section{AUTHOR CONTRIBUTIONS}

YT: conceptualization, data curation, formal analysis, investigation, methodology, visualization, validation, writing - original draft, writing - review and editing

LAD: formal analysis, investigation, methodology, visualization, validation, writing - review and editing

YI: data curation, formal analysis, software

EvO: data curation, formal analysis, software

$\mathrm{JH}$ : formal analysis, investigation, methodology, visualization, validation, writing - review and editing

NT: formal analysis, investigation, methodology, visualization, validation

LA: data curation, formal analysis, investigation

$\mathrm{JZ}$ : data curation, formal analysis, investigation

$\mathrm{MH}$ : data curation, formal analysis, investigation

RD: data curation, formal analysis, investigation

LJ: resources, writing - review and editing

YS: resources, data curation, formal analysis, investigation, writing - review and editing

IK: resources, supervision, software, writing - review and editing

IA: funding acquisition, resources, supervision, validation, writing - review and editing

JB: resources, supervision, validation, writing - review and editing

CS: funding acquisition, conceptualization, project administration, resources, supervision, validation, writing - original draft; writing - review and editing

\section{COMPETING FINANCIAL INTERESTS.}

The authors declare no competing financial interests. 


\section{FIGURE LEGENDS}

Figure 1. Dynamic expression of Plagl1 in Müller glia in the healthy and injured postnatal retina.

a RNAscope in situ hybridization of Plagll (green) and Sox9 (red) riboprobes on P7 wild-type retinal sections. Right side panels are high magnifications of boxed areas.

b RNAseq analysis of P60 Müller glia $\left(\mathrm{GFP}^{+}\right.$cells sorted from GLASTCreERT2;Sun1-sGFP mice) extracted from the database Gene Expression Omnibus with the accession GSE135406, showing Plagll expression along with Müller gli markers (Sox9, Vim, Sox2, Glul, Rlpb), bipolar (Vsx1, $V s x 2)$ and Photoreceptor (Crx) markers in $\mathrm{GFP}^{+}$relative to GFP- cells.

c tSNE-dimensionality reduction to represent retinal cell clustering following NMDA treatment (data extracted from ${ }^{7}$ ). Relative Plagll expression in astrocytes $=0.108$, resting Müller glia $=0.236$ activated Müller glia $=0.308$.

d Temporal profiling of Plagll expression in Müller glia following NMDA or light damage for 3, 6,12 , or $36 \mathrm{H}$ (NMDA) or 4, 16, or $24 \mathrm{H}$ (light damage). RNAseq data extracted from the database Gene Expression Omnibus with the accession GSE135406.

e Immunolabeling of GFAP, Rho, and Arr3 and RNAscope ISH for Plagl1 and Sox 9 on adult wildtype retinal sections 3 days post PBS or MNU treatment.

f Gfap and Plagll expression quantified by RTqPCR in adult retinas treated with PBS or MNU for 3-, 6-, 12-, 24- and 72-hr post MNU. N=4 for all time points. Graph shows mean \pm S.E.M. Unpaired Student t-test, significant differences are indicated $* \mathrm{p}<0.05, * * \mathrm{p}<0.01, * * * \mathrm{p}<0.0001$ *.gcl, ganglion cell layer; inl, inner nuclear layer; onl, outer nuclear layer.

Scale bars $=25 \mu \mathrm{m}$ in a, $50 \mu \mathrm{m}$ in e.

\section{Figure 2. Loss of Plagl1 function perturbs retinal architecture and function.}

a Fundus images (left) and Optical coherence tomography (OCT) derived cross-sectional images (right) of P21 wild type and Plagl1 ${ }^{+/ \text {pat }}$ retinas. Green line indicates the section level. Arrow heads indicate inl disruptions.

b DAPI staining of P21 wild-type and $\mathrm{Plagll}^{+/ \text {pat }}$ retinas, showing retinal ectopias (asterisks) and rosettes (arrowheads). Right side panels are high magnification images of boxed areas. 
c Quantification of rosettes and ectopias in wild-type and $P l a g l 1^{+/ \text {-pat }}$ retinas at P7, P14, P21 and P60. $\mathrm{N}=5$ for all stages.

d Immunostaining for Rhodopsin and Arr3 in P21 wild-type and Plagl1 ${ }^{+/ \text {pat }}$ retinal sections. Arrowheads mark ectopias and rosettes. Blue is DAPI counterstain.

e Immunostaining for OLM marker ZO1 in P21 wild-type and Plagl1 ${ }^{+/ \text {pat }}$ retinal sections. Arrowheads mark gaps in ZO-1 expression at the level of ectopias and rosettes.

f Immunostaining for Iba1 and Cd11b with nuclear DAPI staining of P21 wild-type and Plagl1 ${ }^{+/-}$ ${ }^{\text {pat }}$ retinal sections. Arrowheads mark ectopias and rosettes.

g Staining for Isolectin B4 in P30 wild-type and Plagl1 ${ }^{+\mathrm{m} / \text {-pat }}$ flatmounted retinas. Arrow heads show nodes and tortuous vessels. Right images are high magnifications of the boxed area in left images.

h, i Full field ERG recordings of P30 wild-type and Plagl1 ${ }^{+/ \text {pat }}$ mice under scotopic (h) and photopic (i) conditions. $\mathrm{N}=6$ (wild-type), $\mathrm{N}=5$ (Plagl1 ${ }^{+/ \text {pat }}$ ). Plots show a- and b-wave amplitudes and implicit times. Statistics: means \pm s.e.m. Student $\mathrm{t}$ test. ${ }^{*}, p<0.05 ; * *, p<0.01$; ***, $p<$ 0.005. gcl, ganglion cell layer; inl, inner nuclear layer; onl, outer nuclear layer; olm, outer limiting membrane. Scale bars: $200 \mu \mathrm{m}$ in a (first panel), $25 \mu \mathrm{m}$ in b, d.

\section{Figure 3. Loss of Plagl1 triggers spontaneous reactive gliosis.}

a Immunostaining for vimentin (Vim) in P14 wild-type and $\mathrm{Plagl1}^{+/ \text {pat }}$ retinal sections. Arrow heads point to ectopic Vim upregulation in Plagl1 ${ }^{+/ \text {pat. }}$

b Immunostaining for GFAP in P14, P21 and P45 wild-type and Plagl1 ${ }^{+/ \text {pat }}$ retinal sections. Arrowheads point to ectopic GFAP expression in $\mathrm{Plagll}^{+/ \text {pat }}$ retinas.

c Immunostaining for pERK in P14 wild-type and Plagl1 $^{+/ \text {-pat }}$ retinal sections. Arrowheads point to pERK upregulation in Plagl1 ${ }^{+/ \text {pat }}$ retinas.

d Immunostaining for Glul staining in P14 wild-type and $\mathrm{Plagl1}^{+/ \text {-pat }}$ retinal sections. Arrowheads point to Glul gaps in $\mathrm{Plagll}^{+/ \text {pat }}$ retinas.

e Western blotting for pERK in P14 wild-type and Plagl1 ${ }^{+/ \text {pat }}$ retinas showing increase in pERK protein levels. $\mathrm{N}=6$ for both wild-type and Plagl1 $^{+/ \text {pat }}$ retinas.

f Western blotting for Glul in P14 wild-type and Plagl1 ${ }^{+/ \text {-pat }}$ retinas showing decrease of Glul protein levels. $\mathrm{N}=6$ for both wild-type and $P \operatorname{lag} l 1^{+/ \text {-pat }}$ retinas. 
Touahri et al Plagll sustains Müller glia quiescence

g Schematic of the molecular changes associated with reactive gliosis observed in $P l a g l 1^{+/ \text {pat }}$ retinas.

Statistics: means \pm s.e.m. Student t-test. *, $p<0.05$; **, $p<0.01 ; * * *, p<0.005$. gcl, ganglion cell layer; inl, inner nuclear layer; onl, outer nuclear layer. Scale bars: $25 \mu \mathrm{m}$.

\section{Figure 4. Plagl1 ${ }^{\mathrm{m} / \mathrm{p}}$ Müller glia proliferate ectopically.}

a Immunostaining for Sox9 in P21 wild-type and Plagl ${ }^{+\mathrm{m} / \text {-pat }}$ retinal sections showing increase and mis-localisation of Sox $9^{+}$cells in Plagl1 $^{+\mathrm{m} / \mathrm{p}}$ retinas. Blue is DAPI counterstain.

b Quantification of total, INL, and above INL located Sox $9^{+}$cells number in P21 wild-type and Plagl1 $1^{+\mathrm{m} / \text {-pat }}$ ectopia and rosettes-containing retinal sections. $\mathrm{N}=4$ for wild-type and Plagl1 ${ }^{+/ \text {pat }}$ retinas.

c Immunostaining for Sox9, Pax6, and BrdU on P7 wild-type and Plagll ${ }^{+\mathrm{m} / \text {-pat }}$ retinal sections injected with BrdU for 30 min prior to harvest. Blue is DAPI counterstain. Right images are high magnifications of the boxed area in left images. Arrowheads point to BrdU positive Sox9 cells and asterisks point to BrdU negative for Pax6 in Plagll $^{+/ \text {-pat }}$ retinas.

d Quantification of $\mathrm{BrdU}^{+}$cells, and the percentage of $\mathrm{BrdU}^{+}$cells that are $\mathrm{BrdU}^{+} \mathrm{Sox} 9^{+}$and $\mathrm{BrdU}^{+} \mathrm{Pax}^{+}$in $\mathrm{P} 7$ wild-type and Plagl1 $1^{+m /-p a t}$ normal and rosettes-containing retinal sections injected with BrdU 30 min prior to dissection at P7. wild-type: $\mathrm{N}=5, \operatorname{Plagl1}^{+/ \text {-pat }} \mathrm{N}=3$.

e BrdU birthdating (P7 BrdU to P14 dissection) in vivo, followed by BrdU co-immunostaining with Sox9, Pax6, and Rhodopsin of P14 wild-type and Plagl1 ${ }^{+\mathrm{m} / \text {-pat }}$ retinal sections. Blue is DAPI counterstain.

f Quantification of BrdU birthdating (P7 BrdU to P14 dissection), showing the percentage of $\mathrm{BrdU}^{+}$cells that are $\mathrm{BrdU}^{+} \mathrm{Rho}^{+}, \mathrm{BrdU}^{+} \mathrm{Pax} 6^{+}$and $\mathrm{BrdU}^{+} \mathrm{Sox} 9^{+}$in $\mathrm{P} 14$ wild-type and Plagl1 ${ }^{+m /-p a t}$ normal and rosettes-containing retinal sections in vivo. wild-type: $\mathrm{N}=5, \operatorname{Plagl1}^{+/ \text {-pat }} \mathrm{N}=5$.

Statistics: mean \pm S.E.M, Mann-Whitney U-test. significant differences: ${ }^{*} \mathrm{p}<0.05,{ }^{*} \mathrm{p}<0.01$, $* * * \mathrm{p}<0.0001$. gcl, ganglion cell layer; inl, inner nuclear layer; onl, outer nuclear layer. Scale bars: $25 \mu \mathrm{m}$.

Figure 5. Transcriptomic and epigenomic profiling of $\mathrm{Plagll}^{+\mathrm{m} / \mathrm{p}}$ retinas.

a Heatmap and MAplot showing genes differentially expressed in P7 Plagl1 ${ }^{+\mathrm{m} / \text { pat }}$ retina. 
b RNaseq read coverage of Plagll genomic locus in wild-type and Plagl1 ${ }^{+\mathrm{m} / \text {-pat }}$ showing absence of coverage of exon 2 in Plagl1 $1^{+\mathrm{m} / \text {-pat }}$ indicating that all the sequenced transcripts are of paternal origin.

c Venn diagram identifying the proportion of IGN and all imprinted genes within Plagl $^{+\mathrm{m} / \text {-pat }}$ DEGs.

d GO biological processes and KEGG pathways analysis comparing Plagl1 $^{+\mathrm{m} / \text {-pat }}$ retinal transcriptome to Plagll CHIP-seq, Plagl1 GOF in the cortex, Plagl1 ${ }^{+\mathrm{m} / \text {-pat }}$ MEF transcriptome, and Plagll GOF in N2A cell data sets.

e ATACseq MAplot showing differentially accessible chromatin regions in P7 Plagl1 ${ }^{+\mathrm{m} / \text {-pat }}$ vs wild-type retinas.

f Chromatin accessibility heatmap of genes corresponding to DARs that are differentially expressed in Plagl1 ${ }^{+\mathrm{m} / \text {-pat }}$ retinas.

g Venn diagram identifying Müller glia specific genes that are differentially expressed in Plagl $1^{+\mathrm{m} / \text {-pat }}$, followed by Müller glia specific genes that are up and down-regulated in Plagl $1^{+\mathrm{m} /-}$ ${ }^{\text {pat }}$ retinas.

h Characterisation of Müller glia specific genes that are differentially expressed in Plagl $1^{+\mathrm{m} / \text {-pat }}$ retinas. Enriched GO categories include: transcription factors, Notch signalling, other signalling pathways, translation, and metabolism/catabolism.

Figure 6. Plagl1 inhibits Notch signalling and controls cell cycle genes in P7 Müller glia.

a Venn diagram illustrating transcriptomic comparison of Müller glia after retinal treatment with NMDA/LD and Plagl1 $1^{+\mathrm{m} / \text { pat }}$ retinas showing shared and specific DEGs in the 3 conditions.

b Quantification and classification of shared DEGs in of NMDA/LD-activated Müller glia at different time points and Plagl ${ }^{\mathrm{m} / \text {-pat }}$ retinas that follow opposite transcriptional trajectories (up-in NMDA/LD Müller glia and down in Plagl1 $^{+\mathrm{m} / \text {-pat }}$ and vice versa).

c Violin plot showing increased expression of Notch pathway genes Hes 1, Hes5, Notch1, and Notch2 in P7 Plagl1 ${ }^{+\mathrm{m} / \text { pat }}$ retinas.

d Comparison of Hes1, Hes5, and Notch2 transcript levels in Plagl1 ${ }^{+\mathrm{m} / \text { pat }}$ retinas to NMDA/LD Müller glia at different time points post injury, comparing RNA-seq datasets. 
e RNAscope probe labelling of Hes1, Hes5, and Sox9 on P7 retinal cross sections from wild-type and Plagl1 ${ }^{+\mathrm{m} / \text {-pat }}$ containing rosettes and showing Hes 1 and Hes 5 up-regulation is colocalized with Sox9 staining.

f Heatmap representing cell cycle genes differentially expressed in wild-type vs $P$ lagl $1^{+\mathrm{m} / \text {-pat }}$ retinas.

g Violin plot showing up-regulation of cell cycle genes Cdk6 and Pak4 in P7 Plagl1 ${ }^{\mathrm{m} / \text { pat }}$ retinas compared to wild-type.

\section{Supplementary Figure 1. Plagll expression is down-regulated in Müller glia following injury.}

a,b RNAscope staining for Plagl1, Sox9, and immunofluorescence for GFAP, Rho and Arrestin3 in P7 wild-type retinal section of mice treated with PBS and MNU 7(a) - and 21(b) -days post injection.

c,d Quantification of Plagl1, GFAP and Recovrin expression by qRT-PCR in wild-type retinas treated with PBS and MNU and collected after 7-days (c) and 21-days (d) post injection. $\mathrm{N}=3$ for all time points.

Statistics: mean \pm S.E.M, Student t-test. significant differences: ${ }^{*} \mathrm{p}<0.05,{ }^{*} \mathrm{p}<0.01,{ }^{* * *} \mathrm{p}<0.0001$ gcl, ganglion cell layer; inl, inner nuclear layer; onl, outer nuclear layer. Scale bars: $50 \mu \mathrm{m}$.

\section{Supplementary Figure 2. Plagll is required to sustain retinal integrity.}

a-c DAPI nuclear staining of P7 (a), P14 (b), and P60 (c) wild-type and Plagl1 ${ }^{+/ \text {-pat }}$ retina sections, showing retinal ectopias (asterisks) and rosettes (arrowheads). Right side panels are high magnifications of boxed areas.

gcl, ganglion cell layer; inl, inner nuclear layer; le, lens; onl, outer nuclear layer; re, retina.

\section{Supplementary Figure 3. All retinal cell types are present in $\mathrm{Plagl1}^{+\mathrm{m} / \mathrm{p}}$ retinas.}

a-j Immunofluorescence for retinal cell specific markers S-opsin (cones), Pax6 (amacrine and ganglion cells), Calretinin (amacrine cells), Calbindin (horizontal cells), Brn3a (ganglion cells), M-opsin (cones), Syntaxin (amacrine cells), PKC and Vsx2 (bipolar cells), and Rlbp1 (Müller glia) with DAPI nuclear counter-staining of P21 wild-type and Plagl1 ${ }^{+\mathrm{m} / \text {-pat }}$ retinal sections, showing ectopia (Asterisks) and rosettes (Arrow heads) contain all retinal cell types. At least 3 Plagl $^{+\mathrm{m} / \text {-pat }}$ mice of 2 different litters and their wild-type littermates were analyzed. 
gcl, ganglion cell layer; inl, inner nuclear layer; onl, outer nuclear layer.

\section{Supplementary Figure 4. GO term and KEGG pathways analysis of differentially expressed genes in the Plagl1 $^{+m /-p}$ transcriptome.}

a Gene ontology and KEGG analysis identifying molecular functions, biological processes, cellular compartments and pathways deregulated in P7 Plagl1 ${ }^{+\mathrm{m} /-\mathrm{p}}$ retinas.

b Gene ontology analysis showing the top cellular compartments and molecular functions comparing Plagl1 ${ }^{+\mathrm{m} / \text {-pat }}$ retinal transcriptome to Plagll CHIP-seq, Plagll GOF in the cortex, Plagl ${ }^{+\mathrm{m} / \text {-pat }}$ MEF transcriptome, and Plagll GOF in N2A cell data sets.

c Transcriptomic comparison of Plagl1 $1^{+\mathrm{m} / \text {-pat }}$ DEGs vs Norrin KO DEGs

d Transcriptomic comparison of Plagl1 ${ }^{+\mathrm{m} / \text {-pat }}$ DEGs vs $R d s K O$ DEGs

e Transcriptomic comparison of Plagl1 $1^{+\mathrm{m} / \text { pat }}$ DEGs vs Rd10 KO DEGs

\section{Supplementary Figure 5. Cell cycle gene GO terms and KEGG pathways analysis in Plagl1 $^{+m /-p}$ transcriptome.}

a Transcriptomic comparison of NMDA/LD Müller glia and Plagl1 ${ }^{+\mathrm{m} / \text {-pat }}$ showing shared DEGs that follow the same trajectory, up-regulated, or down-regulated in the 3 conditions at different time points.

b Notch signalling pathway genes Notch1 and Dll1 expression in Müller glia from NMDA/LD treated retinas and Plagl1 ${ }^{+\mathrm{m} / \text {-pat }}$ RNAseq a data set.s

c Expression of Notch genes Serr, Dll1 and Jag2 in P7 Plagl1 ${ }^{+\mathrm{m} / \text { pat }}$ RNAseq data set.

d RT-qPCR expression analysis of Notch genes Hes 1, Hes5 and the gliogenic factors Lhx2, Sox2, Sox9 in P7 Plagl1 ${ }^{+\mathrm{m} / \text {-pat }}$ compared to wild-type.

e Transcriptomic comparison of Müller glia from NMDA/LD treated retinas and Plagl1 ${ }^{\mathrm{m} / \text {-pat }}$ retinas showing expression of genes involved in Müller glia reprograming Myb, Nfib, Nfic, Lhx2, and Tead2.

\section{Supplementary Figure 6. Cell cycle genes deregulated in $\mathrm{P}$ Plagl1 $^{+\mathrm{m} /-\mathrm{p}}$ retinas.}

a Transcriptomic comparison of NMDA/LD Müller glia and Plagl1 ${ }^{+\mathrm{m} / \text {-pat }}$ showing cell cycle genes whose expressions are up-regulated in $P \operatorname{Pagl} 1^{+\mathrm{m} / \text {-pat }}$ while down-regulated in NMDA/LD Müller glia; Cep131, Numal. 
Touahri et al

Plagll sustains Müller glia quiescence

b Transcriptomic comparison of NMDA/LD Müller glia and Plagl1 ${ }^{+\mathrm{m} / \text { pat }}$ showing cell cycle genes whose expressions are down-regulated in $P \operatorname{Pagl} 1^{+\mathrm{m} / \text {-pat }}$ and are initially up-regulated in NMDA/LD Müller glia; Lig4, Rab1 1b, Rps3, Uba3, Zwint

\section{REFERENCES}

1 Wilken, M. S. \& Reh, T. A. Retinal regeneration in birds and mice. Curr Opin Genet Dev 40, 57-64, doi:10.1016/j.gde.2016.05.028 (2016).

2 Franze, K. et al. Muller cells are living optical fibers in the vertebrate retina. Proc Natl Acad Sci U S A 104, 8287-8292, doi:10.1073/pnas.0611180104 (2007).

3 Bringmann, A. et al. Muller cells in the healthy and diseased retina. Prog Retin Eye Res 25, 397-424, doi:10.1016/j.preteyeres.2006.05.003 (2006).

4 Goldman, D. Muller glial cell reprogramming and retina regeneration. Nat Rev Neurosci 15, 431-442, doi:10.1038/nrn3723 (2014).

5 Gallina, D., Todd, L. \& Fischer, A. J. A comparative analysis of Muller glia-mediated regeneration in the vertebrate retina. Exp Eye Res 123, 121-130, doi:10.1016/j.exer.2013.06.019 (2014).

6 Bringmann, A. et al. Cellular signaling and factors involved in Muller cell gliosis: neuroprotective and detrimental effects. Prog Retin Eye Res 28, 423-451, doi:10.1016/j.preteyeres.2009.07.001 (2009).

7 Hoang, T. et al. Gene regulatory networks controlling vertebrate retinal regeneration. Science 370, doi:10.1126/science.abb8598 (2020).

8 Hoang T, W. J., Boyd P, Wang F, Santiago C, Jiang L, Lahne M, Todd LJ, Saez C, Yoo S, Keuthan C, Palazzo I, Squires N, Campbell WA, Jia M, Rajaii F, Payail T, Wang G, Ash J, Fischer AJ, Hyde DR, Qian J, Blackshaw S. Comparative transcriptomic and epigenomic analysis identifies key regulators of injury response and neurogenic competence in retinal glia. bioRxiv, doi: https://doi.org/10.1101/717876 (2020).

9 Wan, J., Zhao, X. F., Vojtek, A. \& Goldman, D. Retinal injury, growth factors, and cytokines converge on beta-catenin and pStat 3 signaling to stimulate retina regeneration. Cell Rep 9, 285-297, doi:10.1016/j.celrep.2014.08.048 (2014).

10 Fischer, A. J., Scott, M. A., Ritchey, E. R. \& Sherwood, P. Mitogen-activated protein kinase-signaling regulates the ability of Muller glia to proliferate and protect retinal neurons against excitotoxicity. Glia 57, 1538-1552, doi:10.1002/glia.20868 (2009).

11 Karl, M. O. et al. Stimulation of neural regeneration in the mouse retina. Proc Natl Acad Sci U S A 105, 19508-19513, doi:10.1073/pnas.0807453105 (2008).

12 Close, J. L., Liu, J., Gumuscu, B. \& Reh, T. A. Epidermal growth factor receptor expression regulates proliferation in the postnatal rat retina. Glia 54, 94-104, doi:10.1002/glia.20361 (2006).

13 Das, A. V. et al. Neural stem cell properties of Muller glia in the mammalian retina: regulation by Notch and Wnt signaling. Dev Biol 299, 283-302, doi:10.1016/j.ydbio.2006.07.029 (2006).

14 Del Debbio, C. B. et al. Notch and Wnt signaling mediated rod photoreceptor regeneration by Muller cells in adult mammalian retina. PLoS One 5, e12425, doi:10.1371/journal.pone.0012425 (2010). 
Touahri et al

Plagll sustains Müller glia quiescence

15 Wan, J., Zheng, H., Xiao, H. L., She, Z. J. \& Zhou, G. M. Sonic hedgehog promotes stem-cell potential of Muller glia in the mammalian retina. Biochem Biophys Res Commun 363, 347-354, doi:10.1016/j.bbrc.2007.08.178 (2007).

16 Bassett, E. A. \& Wallace, V. A. Cell fate determination in the vertebrate retina. Trends Neurosci 35, 565-573, doi:10.1016/j.tins.2012.05.004 (2012).

17 Ramachandran, R., Fausett, B. V. \& Goldman, D. Asclla regulates Muller glia dedifferentiation and retinal regeneration through a Lin-28-dependent, let-7 microRNA signalling pathway. Nat Cell Biol 12, 1101-1107, doi:10.1038/ncb2115 (2010).

18 Ramachandran, R., Zhao, X. F. \& Goldman, D. Asclla/Dkk/beta-catenin signaling pathway is necessary and glycogen synthase kinase-3beta inhibition is sufficient for zebrafish retina regeneration. Proc Natl Acad Sci US A 108, 15858-15863, doi:10.1073/pnas.1107220108 (2011).

19 Fausett, B. V., Gumerson, J. D. \& Goldman, D. The proneural basic helix-loop-helix gene ascl1a is required for retina regeneration. J Neurosci 28, 1109-1117, doi:10.1523/JNEUROSCI.4853-07.2008 (2008).

20 Ueki, Y. et al. Transgenic expression of the proneural transcription factor Ascl1 in Muller glia stimulates retinal regeneration in young mice. Proc Natl Acad Sci U S A 112, 13717 13722, doi:10.1073/pnas.1510595112 (2015).

21 Pollak, J. et al. ASCL1 reprograms mouse Muller glia into neurogenic retinal progenitors. Development 140, 2619-2631, doi:10.1242/dev.091355 (2013).

22 Jorstad, N. L. et al. Stimulation of functional neuronal regeneration from Muller glia in adult mice. Nature, doi:10.1038/nature23283 (2017).

23 Ma, L., Hocking, J. C., Hehr, C. L., Schuurmans, C. \& McFarlane, S. Zac1 promotes a Muller glial cell fate and interferes with retinal ganglion cell differentiation in Xenopus retina. Dev Dyn 236, 192-202, doi:10.1002/dvdy.21002 (2007).

24 Ma, L. et al. Zac1 functions through TGFbetaII to negatively regulate cell number in the developing retina. Neural Dev 2, 11, doi:1749-8104-2-11 [pii]

10.1186/1749-8104-2-11 (2007).

25 de Melo, J., Clark, B. S. \& Blackshaw, S. Multiple intrinsic factors act in concert with Lhx2 to direct retinal gliogenesis. Sci Rep 6, 32757, doi:10.1038/srep32757 (2016).

26 Plasschaert, R. N. \& Bartolomei, M. S. Genomic imprinting in development, growth, behavior and stem cells. Development 141, 1805-1813, doi:10.1242/dev.101428 (2014).

27 Al Adhami, H. et al. A systems-level approach to parental genomic imprinting: the imprinted gene network includes extracellular matrix genes and regulates cell cycle exit and differentiation. Genome research 25, 353-367, doi:10.1101/gr.175919.114 (2015).

28 Zacharek, S. J. et al. Lung stem cell self-renewal relies on BMI1-dependent control of expression at imprinted loci. Cell Stem Cell 9, 272-281, doi:10.1016/j.stem.2011.07.007 (2011).

29 Ferron, S. R. et al. Postnatal loss of Dlk1 imprinting in stem cells and niche astrocytes regulates neurogenesis. Nature 475, 381-385, doi:10.1038/nature10229 (2011).

30 Furutachi, S., Matsumoto, A., Nakayama, K. I. \& Gotoh, Y. p57 controls adult neural stem cell quiescence and modulates the pace of lifelong neurogenesis. EMBO J 32, 970981, doi:10.1038/emboj.2013.50 (2013).

31 Varrault, A. et al. Zac1 regulates an imprinted gene network critically involved in the control of embryonic growth. Dev Cell 11, 711-722, doi:10.1016/j.devcel.2006.09.003 (2006). 
Touahri et al

Plagll sustains Müller glia quiescence

32 Young, R. W. Cell differentiation in the retina of the mouse. The Anatomical record 212, 199-205, doi:10.1002/ar.1092120215 (1985).

33 Wang, X., Shu, Q., Ni, Y. \& Xu, G. CRISPR-mediated SOX9 knockout inhibits GFAP expression in retinal glial (Muller) cells. Neuroreport 29, 1504-1508, doi:10.1097/WNR.0000000000001143 (2018).

34 Chen, Y. Y., Liu, S. L., Hu, D. P., Xing, Y. Q. \& Shen, Y. N -methyl- N -nitrosoureainduced retinal degeneration in mice. Exp Eye Res 121, 102-113, doi:10.1016/j.exer.2013.12.019 (2014).

35 Nomura-Komoike, K., Saitoh, F., Komoike, Y. \& Fujieda, H. DNA Damage Response in Proliferating Muller Glia in the Mammalian Retina. Invest Ophthalmol Vis Sci 57, 11691182, doi:10.1167/iovs.15-18101 (2016).

36 Hiura, H., Obata, Y., Komiyama, J., Shirai, M. \& Kono, T. Oocyte growth-dependent progression of maternal imprinting in mice. Genes Cells 11, 353-361, doi:10.1111/j.1365-2443.2006.00943.x (2006).

37 Prusky, G. T., Alam, N. M., Beekman, S. \& Douglas, R. M. Rapid quantification of adult and developing mouse spatial vision using a virtual optomotor system. Invest Ophthalmol Vis Sci 45, 4611-4616, doi:10.1167/iovs.04-0541 (2004).

38 Byrne, L. C. et al. AAV-mediated, optogenetic ablation of Muller Glia leads to structural and functional changes in the mouse retina. PloS one 8, e76075, doi:10.1371/journal.pone.0076075 (2013).

39 MacDonald, R. B. et al. Muller glia provide essential tensile strength to the developing retina. J Cell Biol 210, 1075-1083, doi:10.1083/jcb.201503115 (2015).

40 Damiani, D. et al. Dicer inactivation leads to progressive functional and structural degeneration of the mouse retina. J Neurosci 28, 4878-4887, doi:10.1523/JNEUROSCI.0828-08.2008 (2008).

41 Reichenbach, A. \& Bringmann, A. New functions of Muller cells. Glia 61, 651-678, doi:10.1002/glia.22477 (2013).

42 Lahne, M., Li, J., Marton, R. M. \& Hyde, D. R. Actin-Cytoskeleton- and Rock-Mediated INM Are Required for Photoreceptor Regeneration in the Adult Zebrafish Retina. $J$ Neurosci 35, 15612-15634, doi:10.1523/JNEUROSCI.5005-14.2015 (2015).

43 Poche, R. A., Furuta, Y., Chaboissier, M. C., Schedl, A. \& Behringer, R. R. Sox9 is expressed in mouse multipotent retinal progenitor cells and functions in Muller glial cell development. J Comp Neurol 510, 237-250, doi:10.1002/cne.21746 (2008).

44 Marquardt, T. et al. Pax6 is required for the multipotent state of retinal progenitor cells. Cell 105, 43-55, doi:S0092-8674(01)00295-1 [pii] (2001).

45 Huang, S. M. \& Stallcup, M. R. Mouse Zac1, a transcriptional coactivator and repressor for nuclear receptors. Mol Cell Biol 20, 1855-1867 (2000).

46 Huang, S. M., Schonthal, A. H. \& Stallcup, M. R. Enhancement of p53-dependent gene activation by the transcriptional coactivator Zac1. Oncogene 20, 2134-2143 (2001).

47 Rozenfeld-Granot, G. et al. A positive feedback mechanism in the transcriptional activation of Apaf-1 by p53 and the coactivator Zac-1. Oncogene 21, 1469-1476 (2002).

48 Hoffmann, A. et al. Transcriptional activities of the zinc finger protein Zac are differentially controlled by DNA binding. Mol Cell Biol 23, 988-1003 (2003).

49 Varrault, A. et al. Identification of Plagl1/Zac1 binding sites and target genes establishes its role in the regulation of extracellular matrix genes and the imprinted gene network. Nucleic Acids Res 45, 10466-10480, doi:10.1093/nar/gkx672 (2017). 
Touahri et al

Plagll sustains Müller glia quiescence

50 Abdollahi, A. LOT1 (ZAC1/PLAGL1) and its family members: mechanisms and functions. J Cell Physiol 210, 16-25 (2007).

51 Spengler, D. et al. Regulation of apoptosis and cell cycle arrest by Zac1, a novel zinc finger protein expressed in the pituitary gland and the brain. Embo J 16, 2814-2825 (1997).

52 Varrault, A. et al. hZAC encodes a zinc finger protein with antiproliferative properties and maps to a chromosomal region frequently lost in cancer. Proc Natl Acad Sci US A 95, 8835-8840 (1998).

53 Bilanges, B. et al. Alternative splicing of the imprinted candidate tumor suppressor gene ZAC regulates its antiproliferative and DNA binding activities. Oncogene 20, 1246-1253 (2001).

54 Adnani, L. et al. Zac1 Regulates the Differentiation and Migration of Neocortical Neurons via Pac1. J Neurosci 35, 13430-13447, doi:10.1523/JNEUROSCI.0777-15.2015 (2015).

55 Rraklli, V., Sodersten, E., Nyman, U., Hagey, D. W. \& Holmberg, J. Elevated levels of ZAC1 disrupt neurogenesis and promote rapid in vivo reprogramming. Stem Cell Res 16, 1-9, doi:10.1016/j.scr.2015.11.002 (2016).

56 Buenrostro, J. D., Wu, B., Chang, H. Y. \& Greenleaf, W. J. ATAC-seq: A Method for Assaying Chromatin Accessibility Genome-Wide. Curr Protoc Mol Biol 109, 2129 212129 29, doi:10.1002/0471142727.mb2129s109 (2015).

57 Jones, B. W. \& Marc, R. E. Retinal remodeling during retinal degeneration. Exp Eye Res 81, 123-137, doi:10.1016/j.exer.2005.03.006 (2005).

58 Heng, J. S. et al. Hypoxia tolerance in the Norrin-deficient retina and the chronically hypoxic brain studied at single-cell resolution. Proc Natl Acad Sci U S A 116, 9103-9114, doi:10.1073/pnas.1821122116 (2019).

59 Wang, Y., Rhee, K. D., Pellegrini, M. \& Yang, X. J. Impacts of ciliary neurotrophic factor on the retinal transcriptome in a mouse model of photoreceptor degeneration. Sci Rep 10, 6593, doi:10.1038/s41598-020-63519-1 (2020).

60 Clark, B. S. et al. Single-Cell RNA-Seq Analysis of Retinal Development Identifies NFI Factors as Regulating Mitotic Exit and Late-Born Cell Specification. Neuron 102, 1111 1126 e1115, doi:10.1016/j.neuron.2019.04.010 (2019).

61 Muto, A., Iida, A., Satoh, S. \& Watanabe, S. The group E Sox genes Sox8 and Sox9 are regulated by Notch signaling and are required for Muller glial cell development in mouse retina. Exp Eye Res 89, 549-558, doi:10.1016/j.exer.2009.05.006 (2009).

62 Roesch, K. et al. The transcriptome of retinal Muller glial cells. J Comp Neurol 509, $225-$ 238, doi:10.1002/cne.21730 (2008).

63 Powner, M. B. et al. Perifoveal muller cell depletion in a case of macular telangiectasia type 2. Ophthalmology 117, 2407-2416, doi:10.1016/j.ophtha.2010.04.001 (2010).

64 Pagotto, U. et al. The expression of the antiproliferative gene ZAC is lost or highly reduced in nonfunctioning pituitary adenomas. Cancer Res 60, 6794-6799 (2000).

65 Koy, S. et al. Loss of expression of ZAC/LOT1 in squamous cell carcinomas of head and neck. Head Neck 26, 338-344 (2004).

66 Abdollahi, A., Gruver, B. N., Patriotis, C. \& Hamilton, T. C. Identification of epidermal growth factor-responsive genes in normal rat ovarian surface epithelial cells. Biochem Biophys Res Commun 307, 188-197 (2003). 
Touahri et al

Plagll sustains Müller glia quiescence

67 Abdollahi, A. et al. Identification of a gene containing zinc-finger motifs based on lost expression in malignantly transformed rat ovarian surface epithelial cells. Cancer Res $\mathbf{5 7}$, 2029-2034 (1997).

68 Wohl, S. G., Jorstad, N. L., Levine, E. M. \& Reh, T. A. Muller glial microRNAs are required for the maintenance of glial homeostasis and retinal architecture. Nat Commun 8, 1603, doi:10.1038/s41467-017-01624-y (2017).

69 Rueda, E. M. et al. The Hippo Pathway Blocks Mammalian Retinal Muller Glial Cell Reprogramming. Cell Rep 27, 1637-1649 e1636, doi:10.1016/j.celrep.2019.04.047 (2019).

70 Dyer, M. A. \& Cepko, C. L. Control of Muller glial cell proliferation and activation following retinal injury. Nat Neurosci 3, 873-880, doi:10.1038/78774 (2000).

71 Hamon, A. et al. Linking YAP to Muller Glia Quiescence Exit in the Degenerative Retina. Cell Rep 27, 1712-1725 e1716, doi:10.1016/j.celrep.2019.04.045 (2019).

72 de Melo, J. et al. Injury-independent induction of reactive gliosis in retina by loss of function of the LIM homeodomain transcription factor Lhx2. Proc Natl Acad Sci US A 109, 4657-4662, doi:10.1073/pnas.1107488109 (2012).

73 Barlow, D. P. \& Bartolomei, M. S. Genomic imprinting in mammals. Cold Spring Harbor perspectives in biology 6, doi:10.1101/cshperspect.a018382 (2014).

74 Wilkins, J. F., Ubeda, F. \& Van Cleve, J. The evolving landscape of imprinted genes in humans and mice: Conflict among alleles, genes, tissues, and kin. BioEssays : news and reviews in molecular, cellular and developmental biology 38, 482-489, doi:10.1002/bies.201500198 (2016).

75 Patten, M. M., Cowley, M., Oakey, R. J. \& Feil, R. Regulatory links between imprinted genes: evolutionary predictions and consequences. Proceedings. Biological sciences 283, doi:10.1098/rspb.2015.2760 (2016).

76 Ferron, S. R. et al. Differential genomic imprinting regulates paracrine and autocrine roles of IGF2 in mouse adult neurogenesis. Nat Commun 6, 8265, doi:10.1038/ncomms9265 (2015).

77 Falix, F. A., Aronson, D. C., Lamers, W. H. \& Gaemers, I. C. Possible roles of DLK1 in the Notch pathway during development and disease. Biochim Biophys Acta 1822, 988995, doi:10.1016/j.bbadis.2012.02.003 (2012).

78 Kim, K. A., Kim, J. H., Wang, Y. \& Sul, H. S. Pref-1 (preadipocyte factor 1) activates the MEK/extracellular signal-regulated kinase pathway to inhibit adipocyte differentiation. Mol Cell Biol 27, 2294-2308, doi:10.1128/MCB.02207-06 (2007).

79 Pinilla, I., Lund, R. D. \& Sauve, Y. Contribution of rod and cone pathways to the darkadapted electroretinogram (ERG) b-wave following retinal degeneration in RCS rats. Vision Res 44, 2467-2474, doi:10.1016/j.visres.2004.05.020

S0042-6989(04)00266-4 [pii] (2004).

80 Cantrup, R. et al. Cell-type specific roles for PTEN in establishing a functional retinal architecture. PLoS One 7, e32795, doi:10.1371/journal.pone.0032795 (2012). 
a

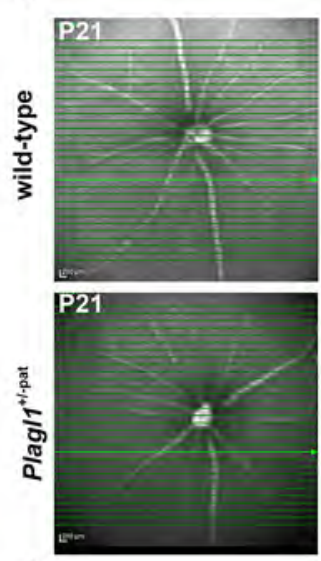

d

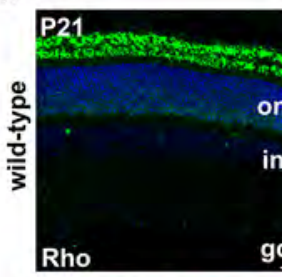

$\frac{1}{0}$

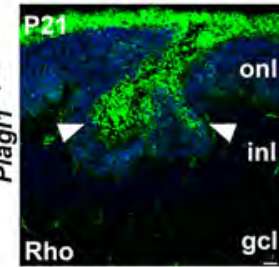

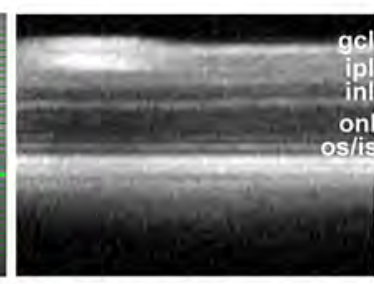
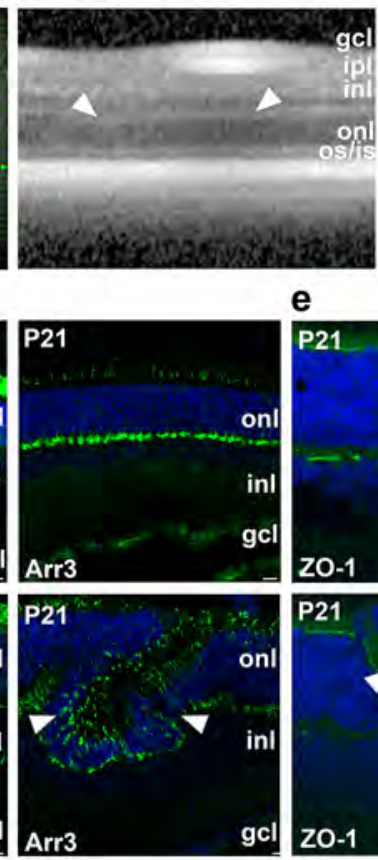

e b
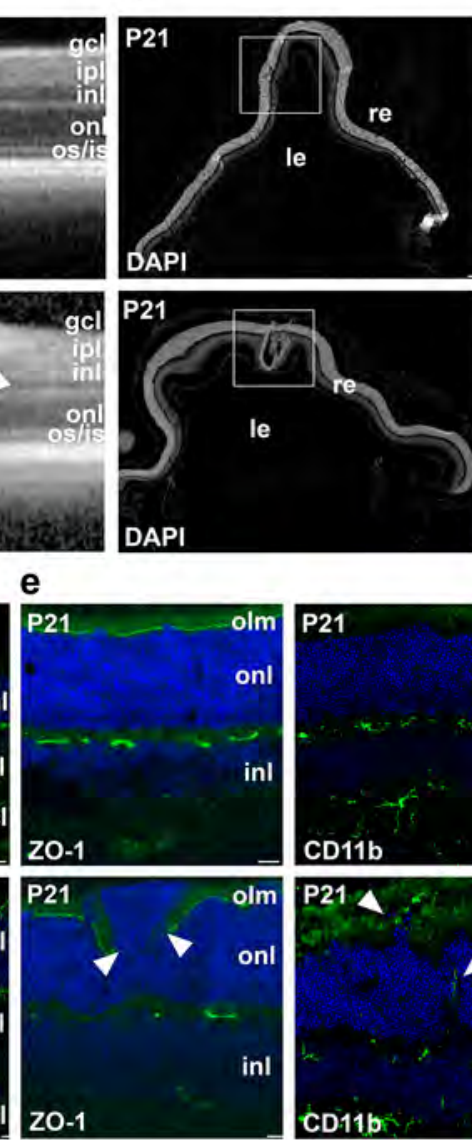

C
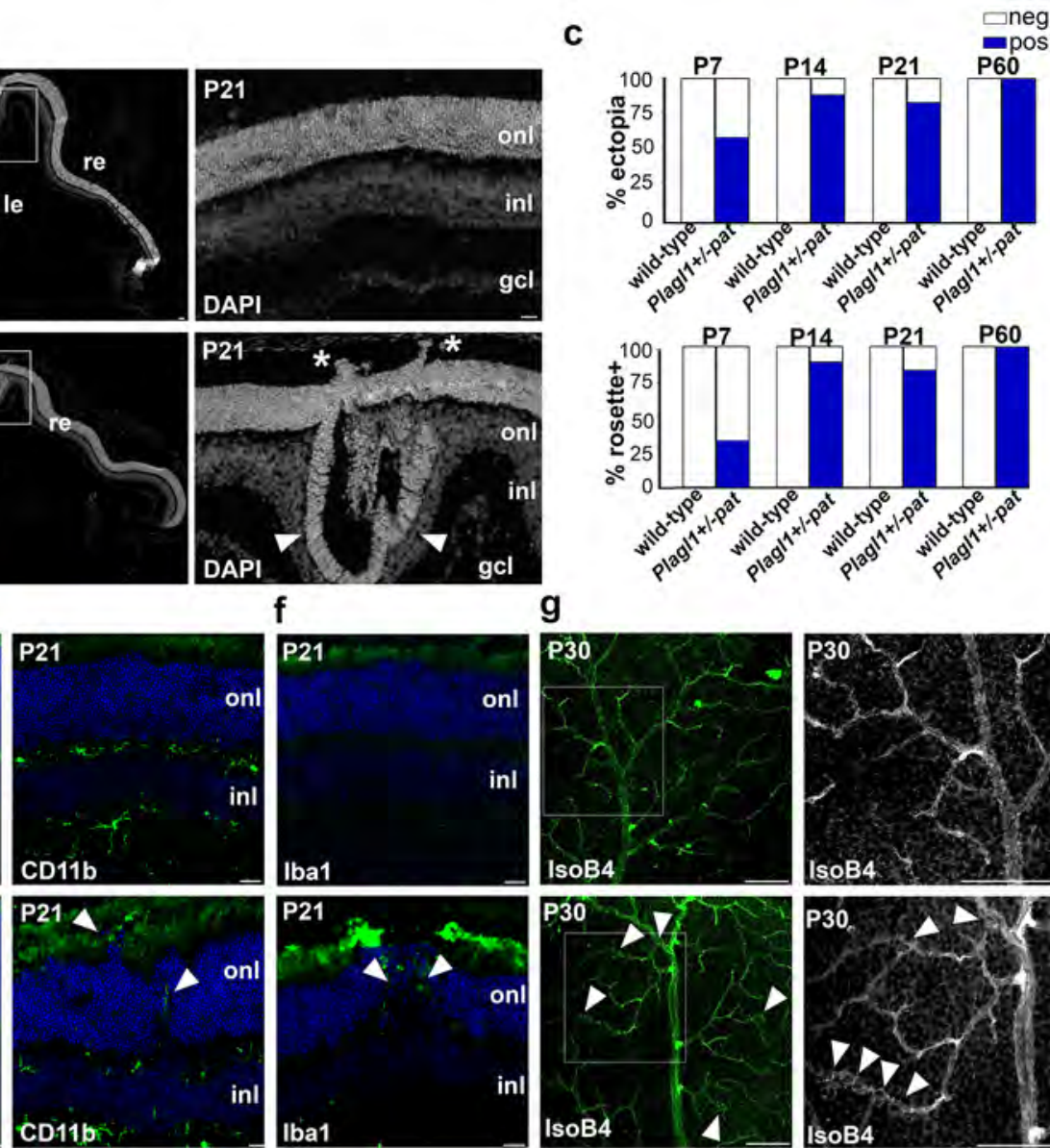
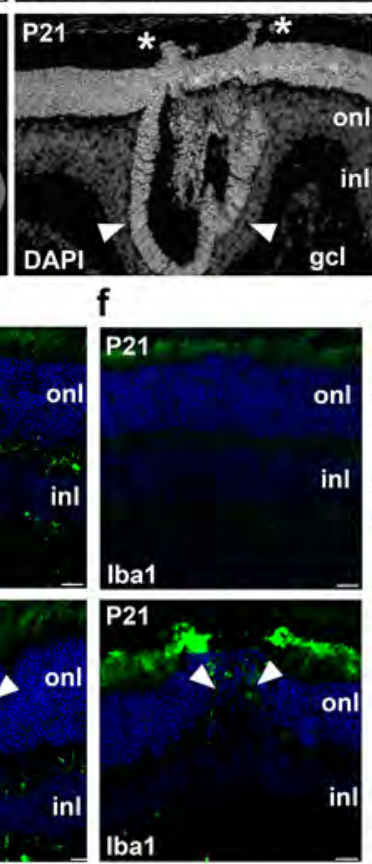



g
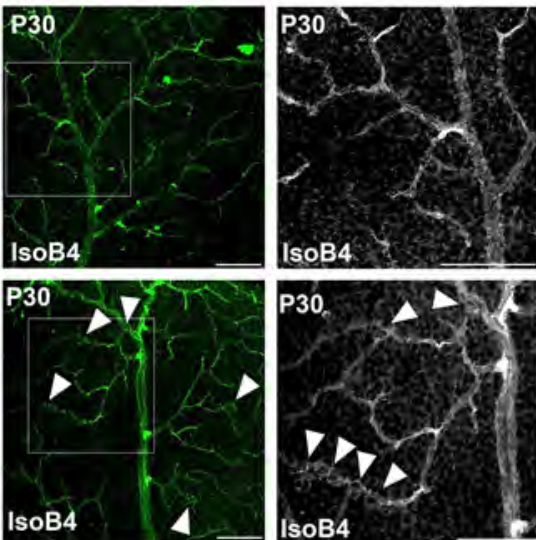

h Scotopic intensity response

- wild-type
- Plaglitit-pat

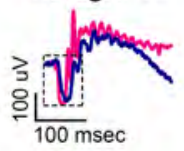

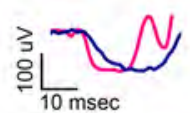


Photopic intensity response
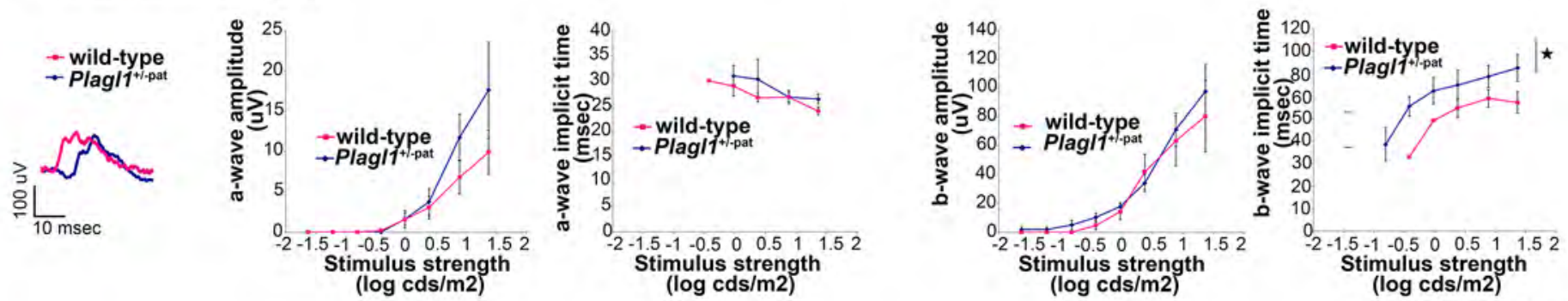

Touahri et al. Figure 2 


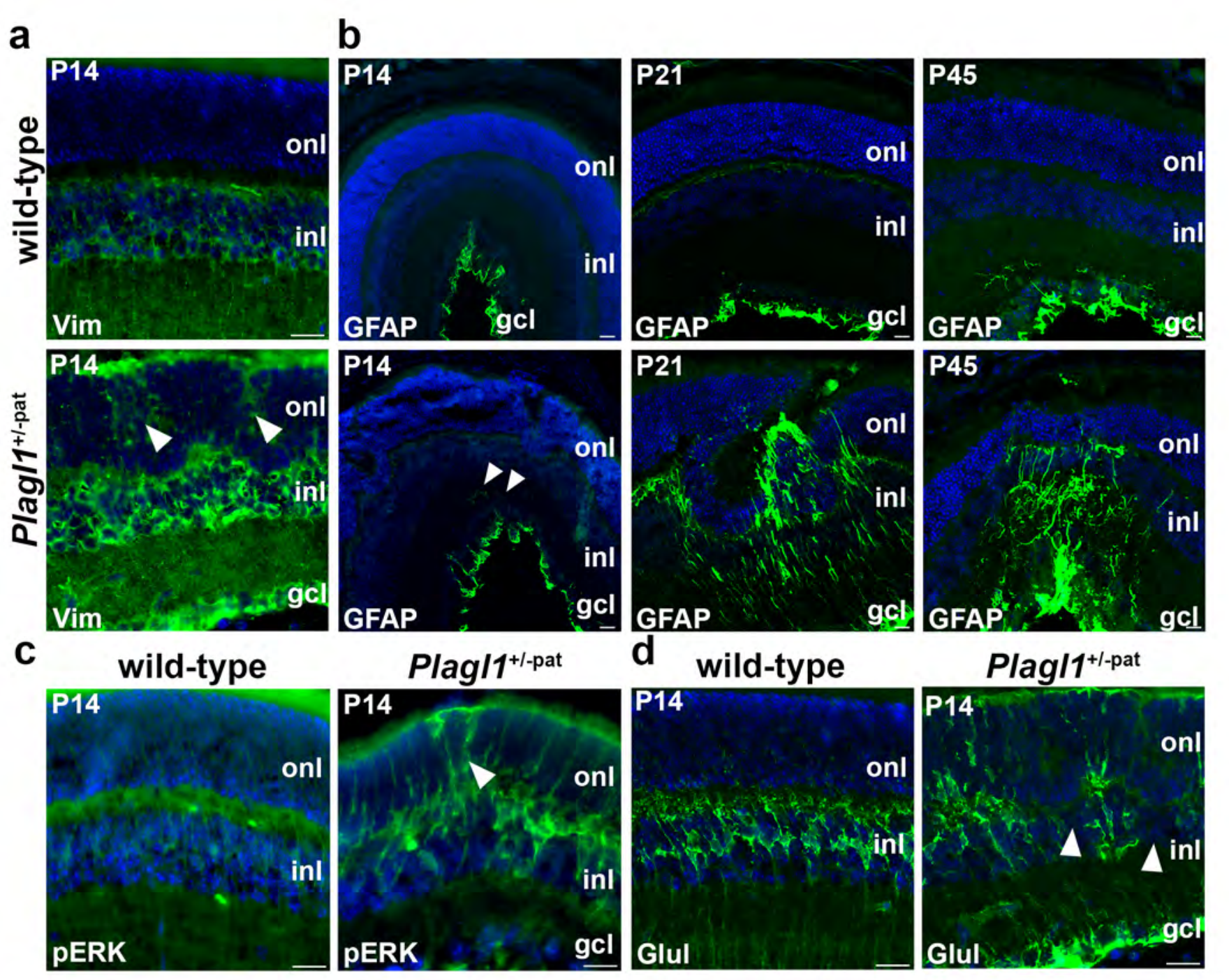

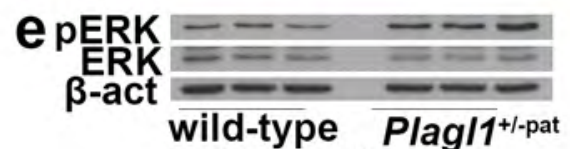

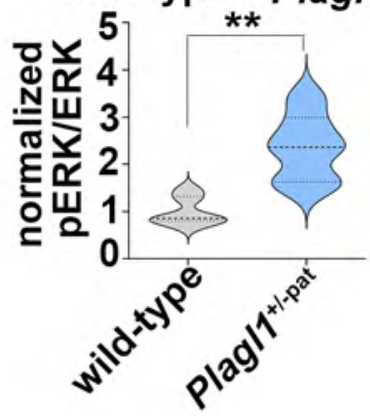

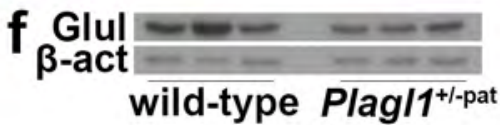

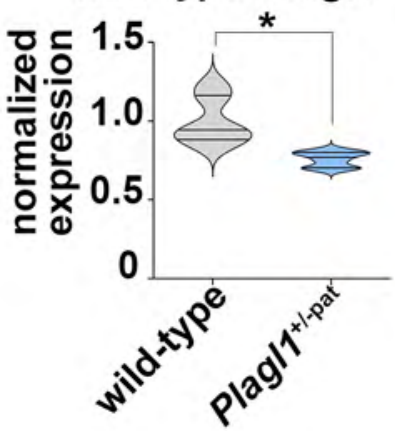

g

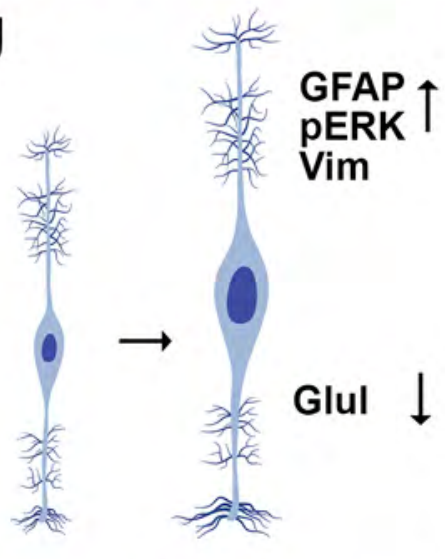

Touahri et al. Figure 3 
a

wild-type

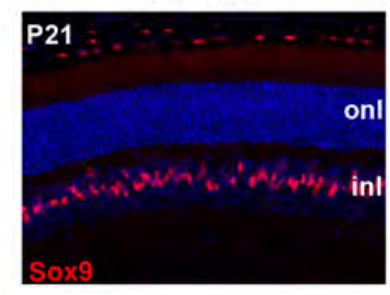

C
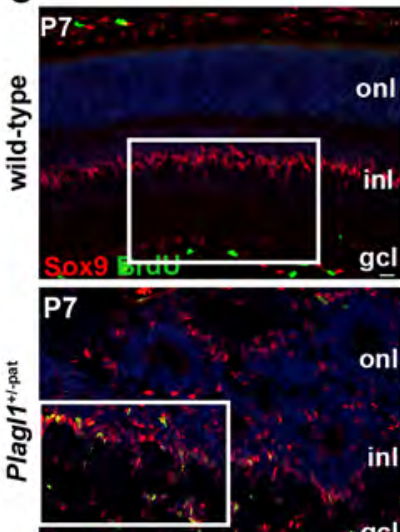

Soxts BrdU
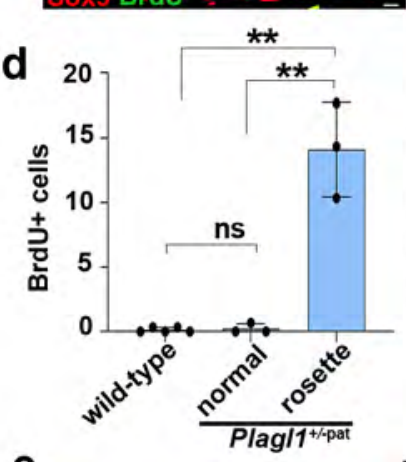

6

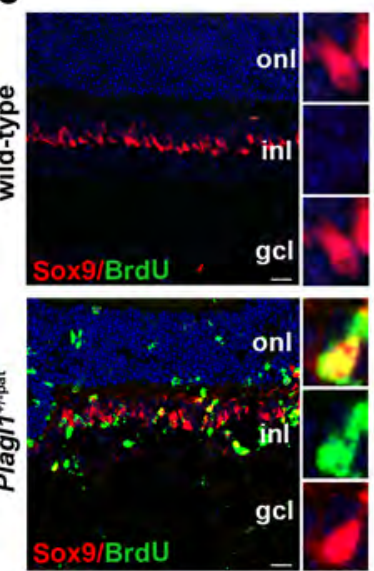

Plag/1 1/-pat

ectopia

rosette

wild-type
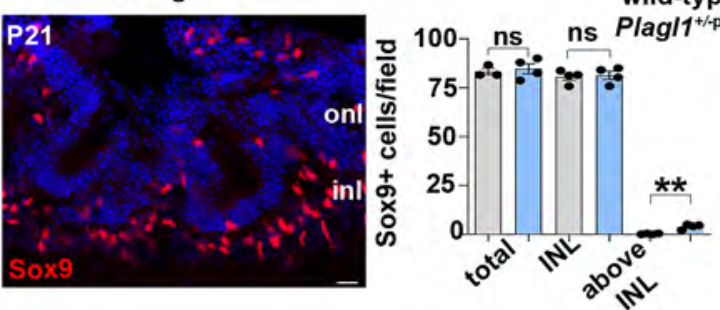

*** wild-type

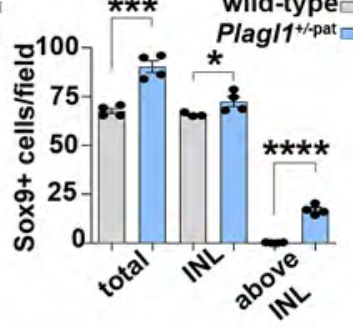

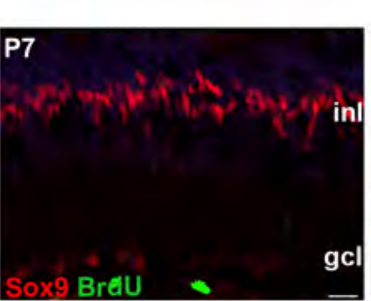
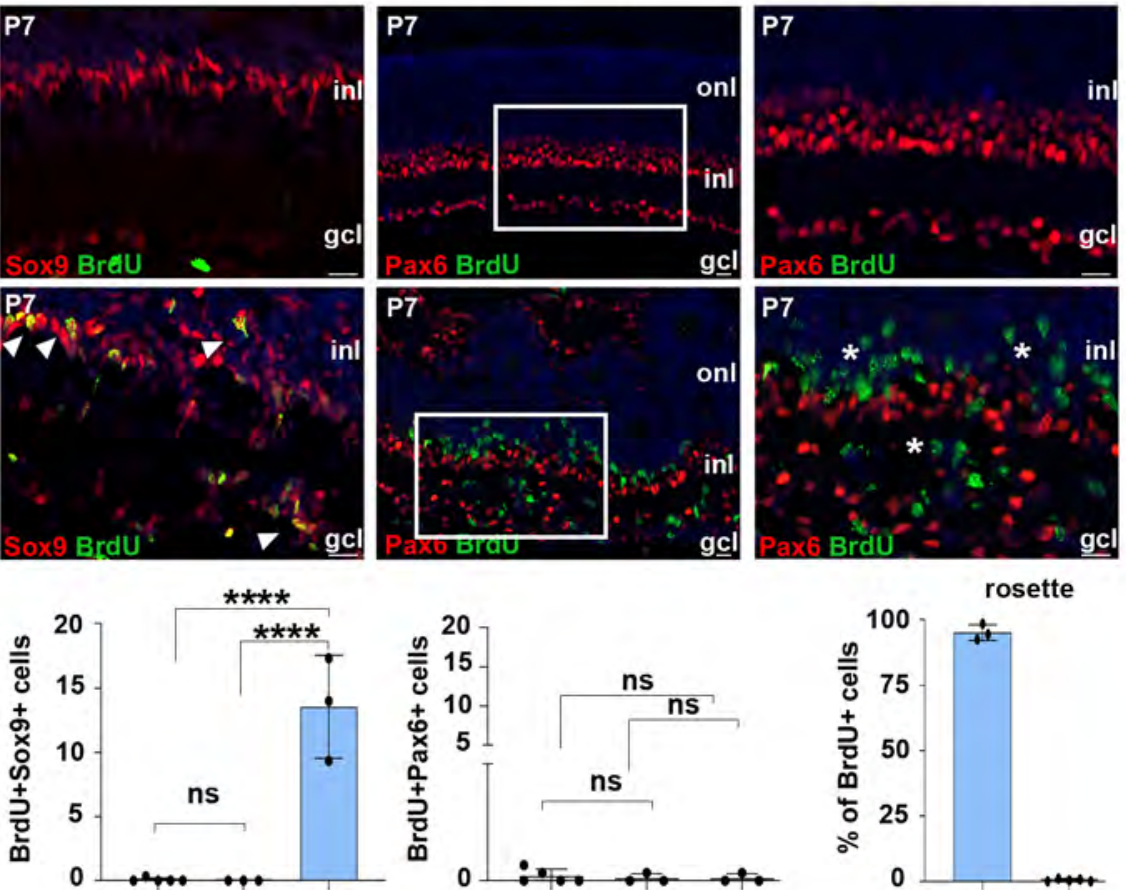

$\star * \star *$

rosette
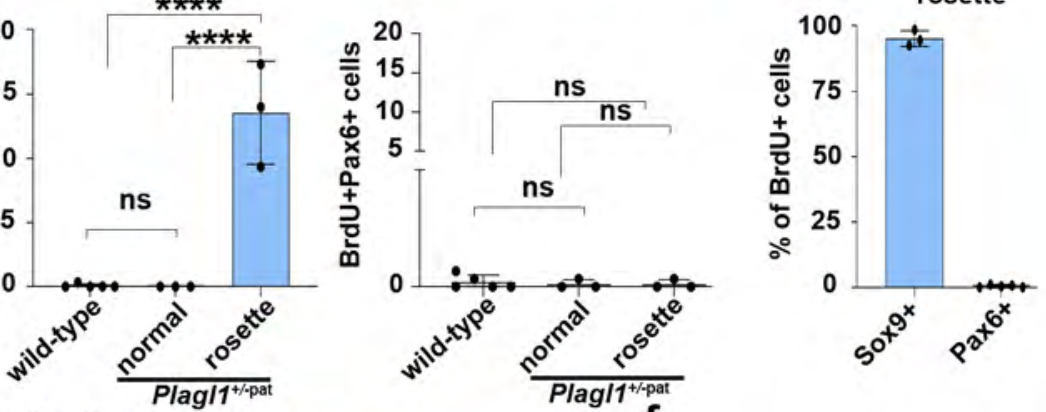

BrdU birthdating P7 $\rightarrow$ P14

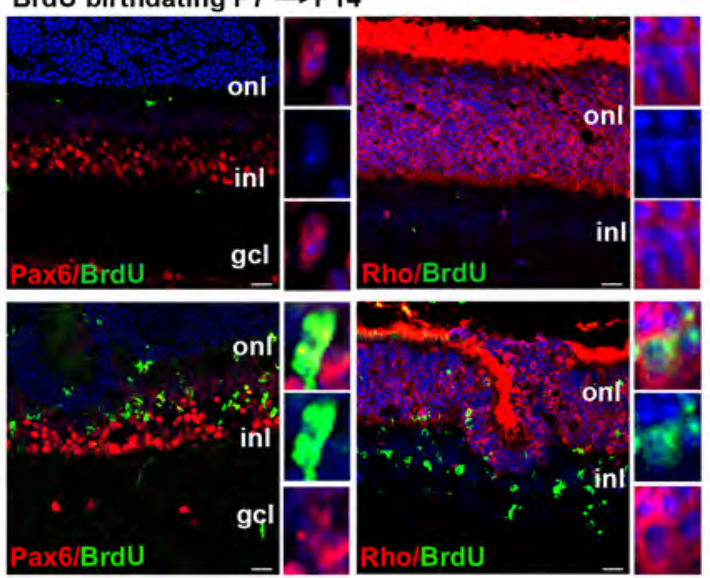

Plagl1 ${ }^{\text {t/pat }} \square$






\section{a}

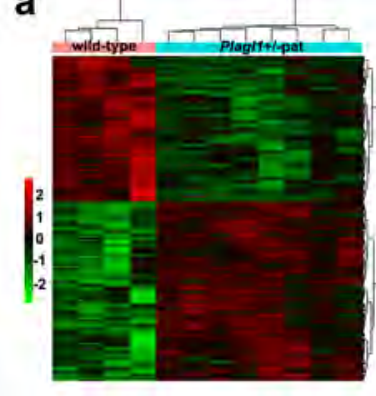

d


g

MG specific genes DEG in Plag/1 ${ }^{\text {+-pat }}$



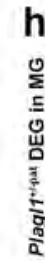
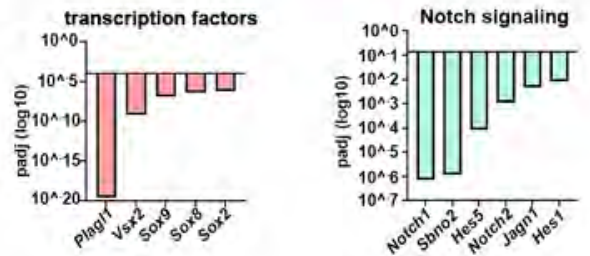

b

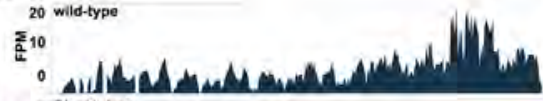
$\sum_{10}^{20}$

(1)

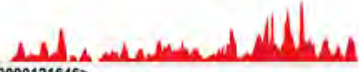
Plagit:ENSMUSTO0000121646>

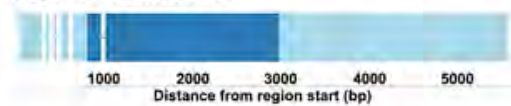

C Plag/1/1/pat DEG/Plag/1 IGN

Plag/1+1-pat DEG/total imprinted genes
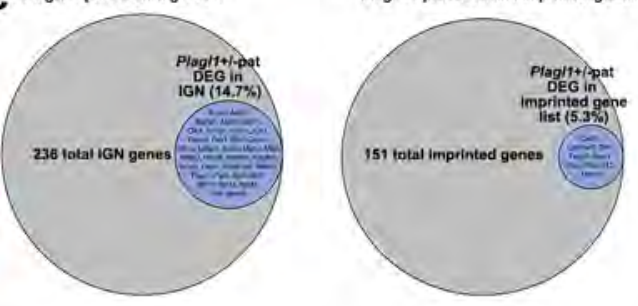

e

Heatmap KEGG pattwways



f
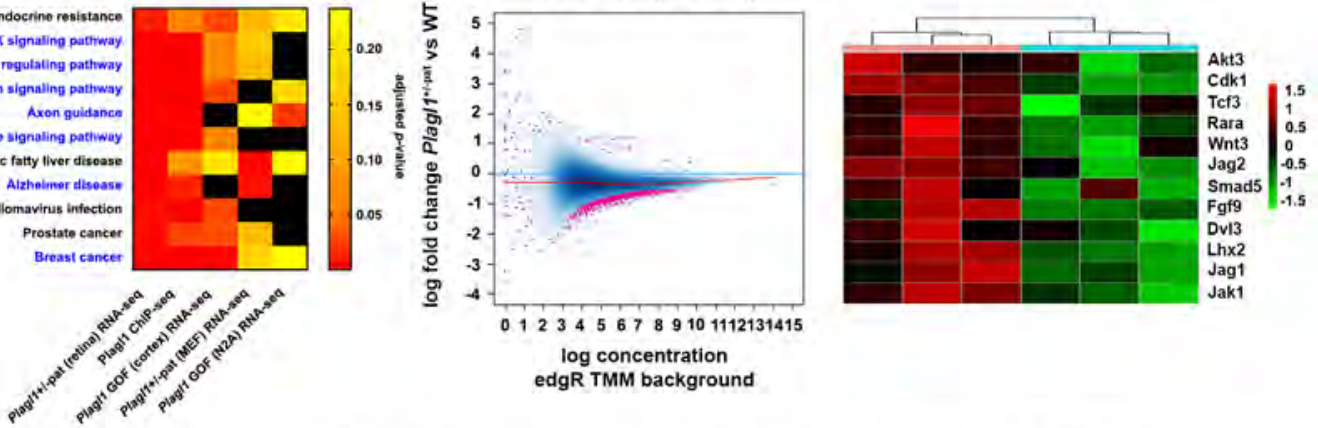

MG specific genes up-regulated in Plagi $7^{+ \text {;pen }}$



MG specific genes down-regulated in Plagl1*ipat
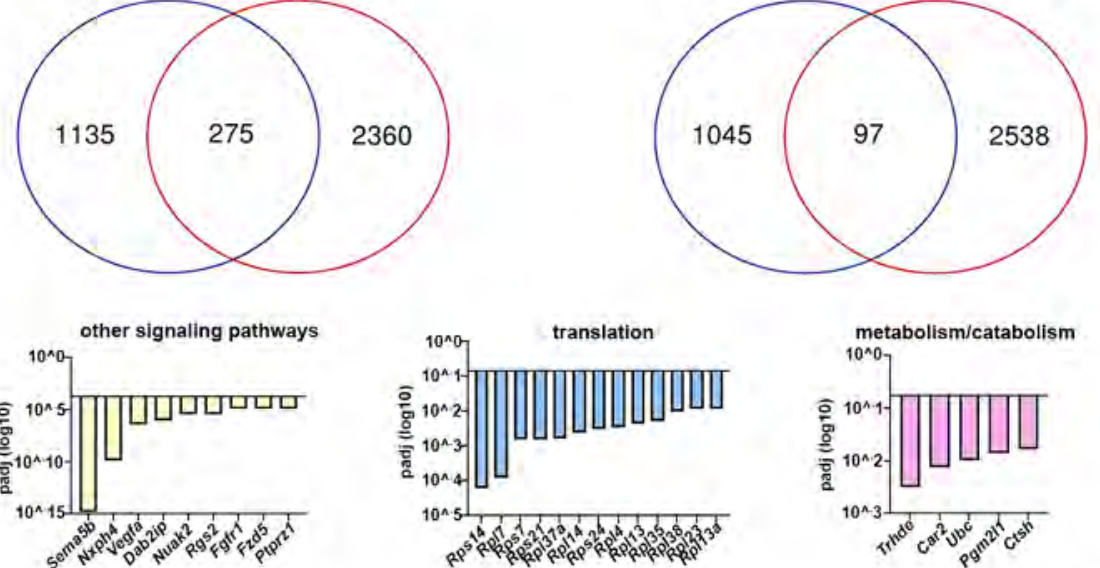

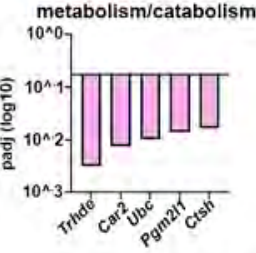

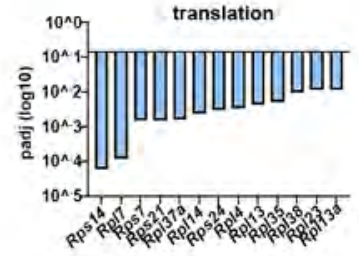

Touahri et al. Figure 5 
a Plagl1+pot

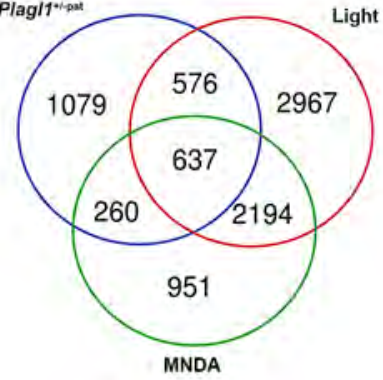

C

Hes1
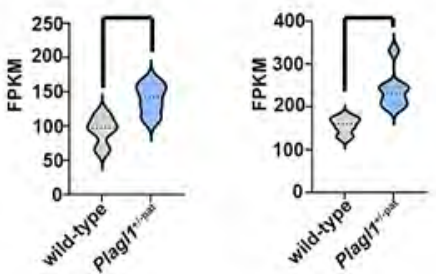
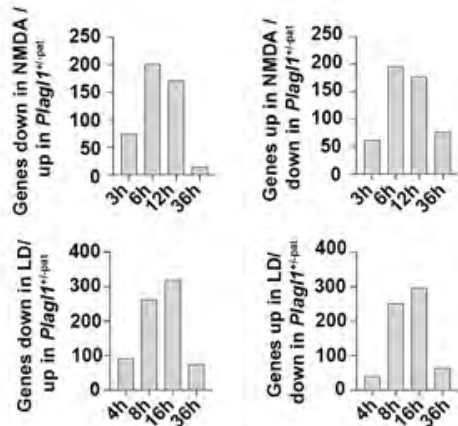

Notch1
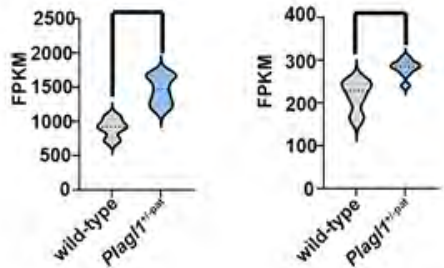

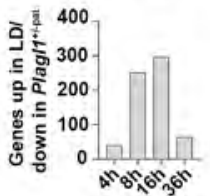

Notch2 d

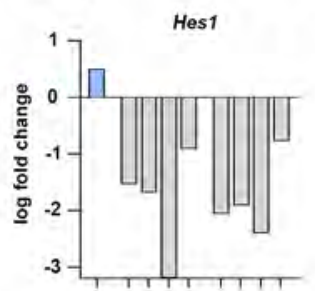

,

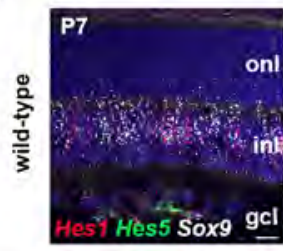

$\frac{1}{\frac{8}{4}}$

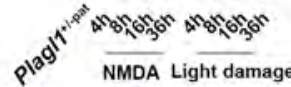

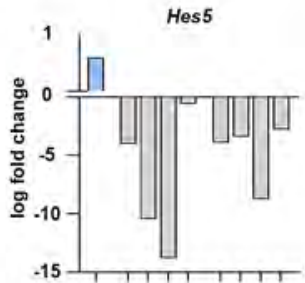

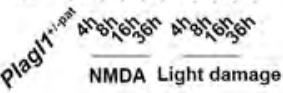

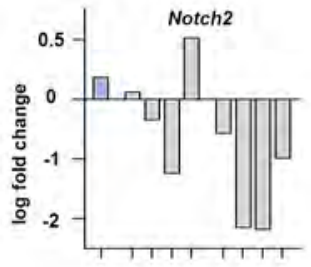
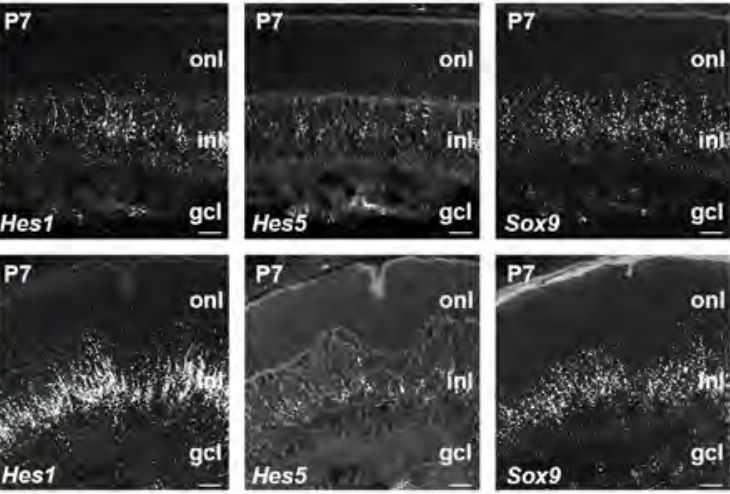

g


Touahri et al. Figure 6 
$a$

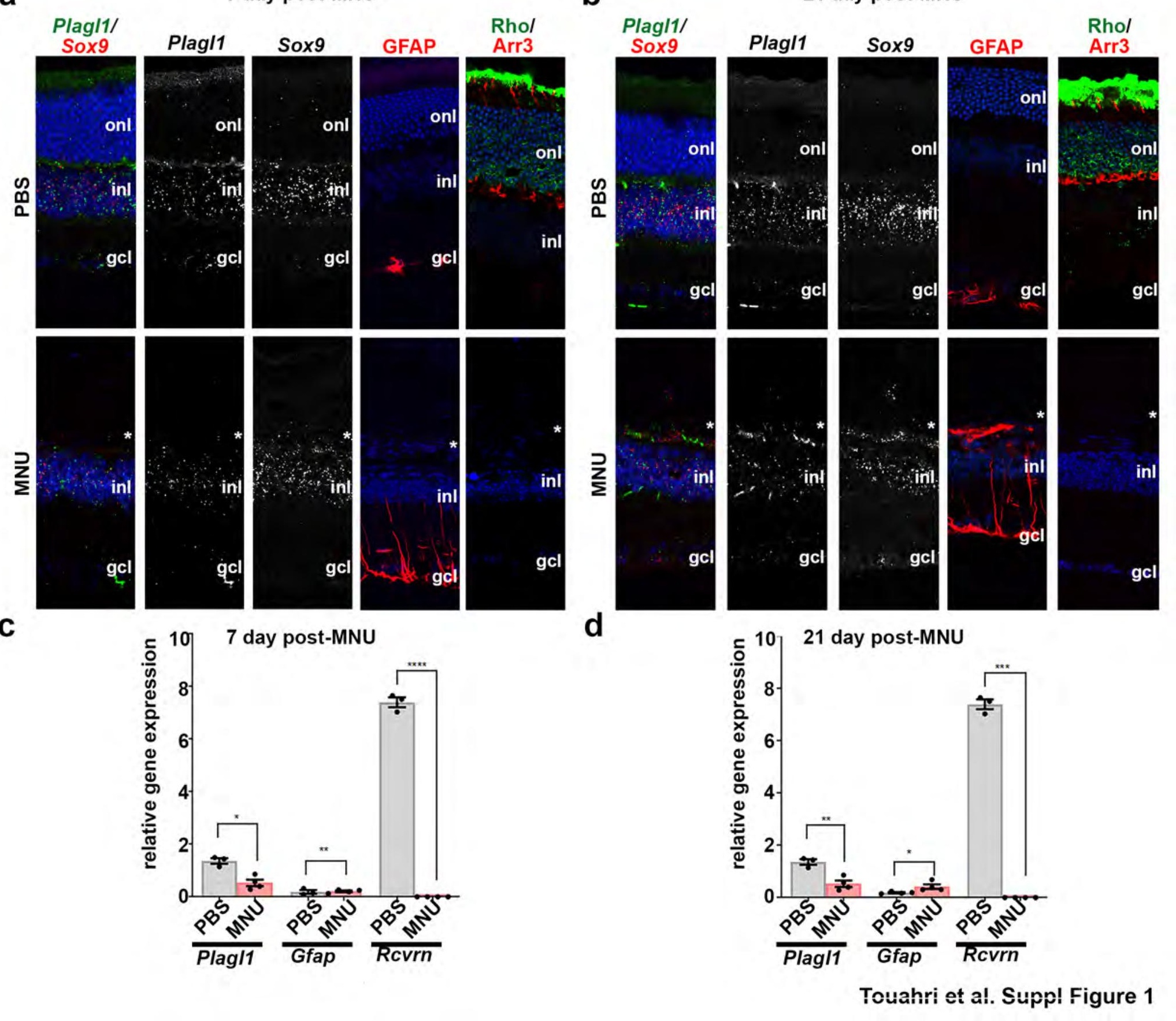




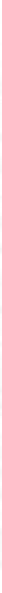




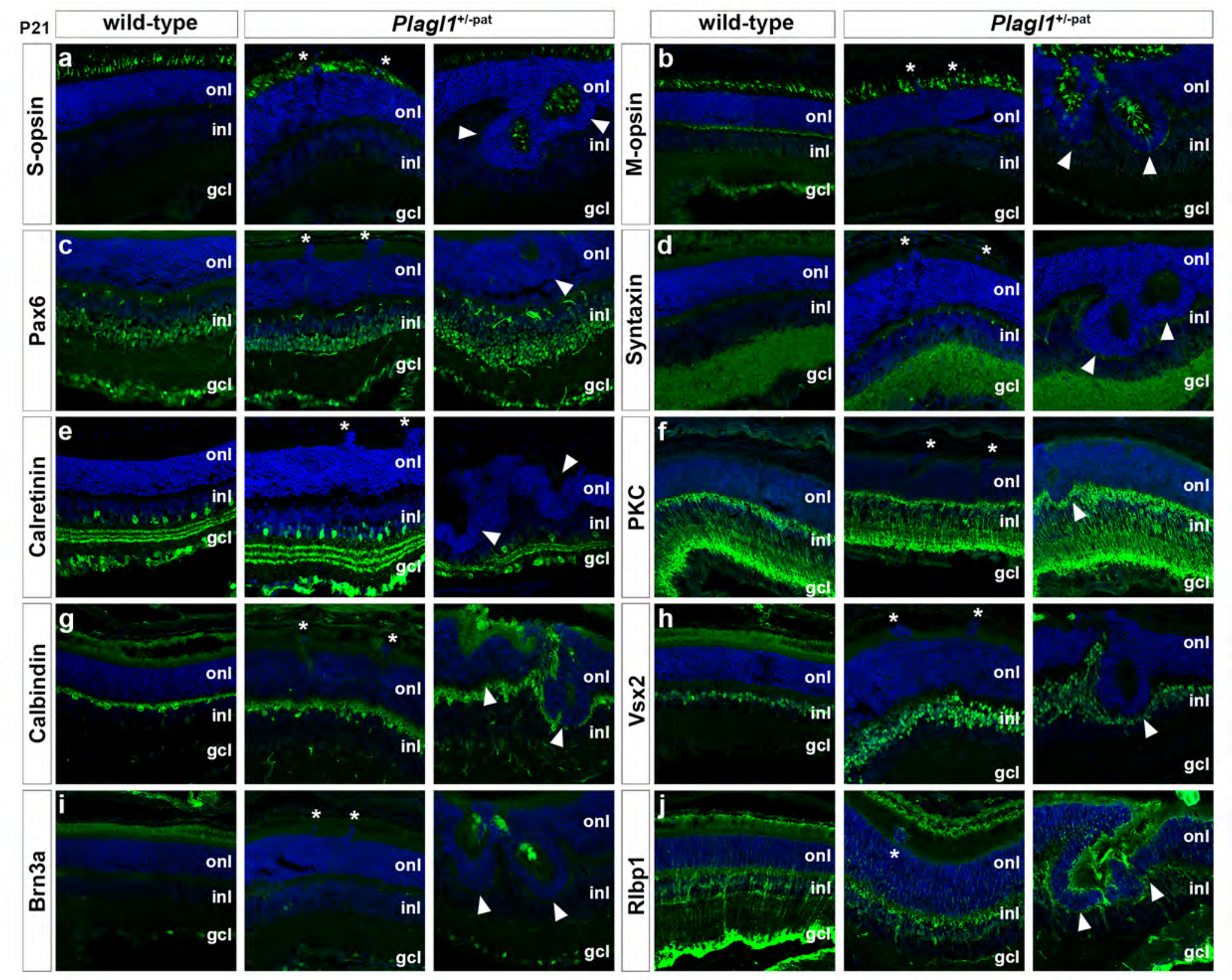

Touahri et al. Suppl. Figure 3 
a

wild-type



C
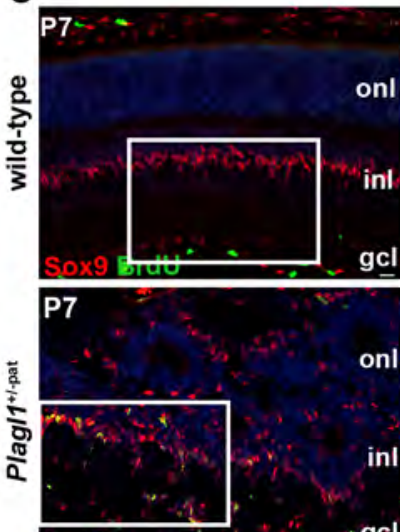

Soxts BrdU
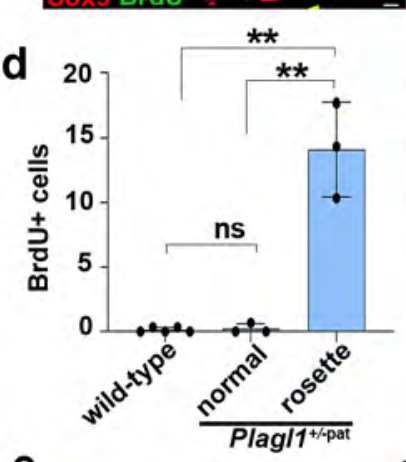

6

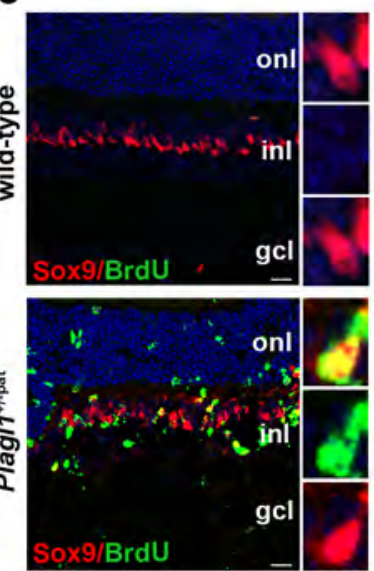

Plag/1 1/-pat

ectopia

rosette

wild-type
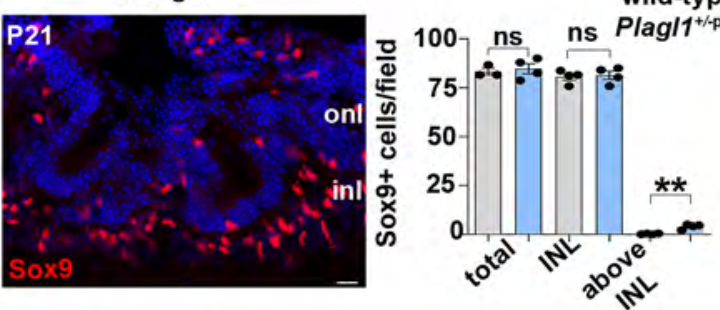

*** wild-type

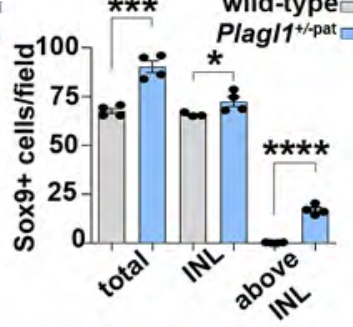

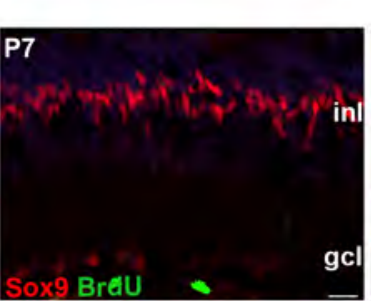
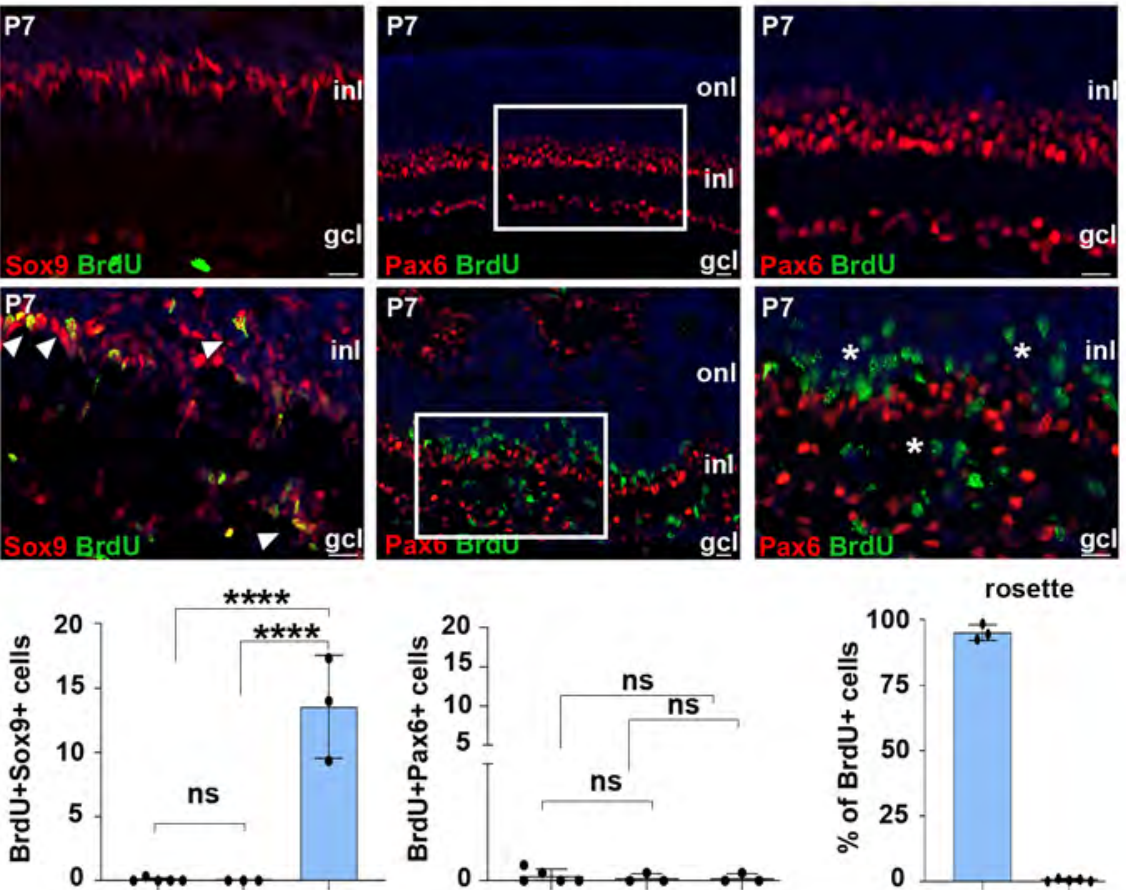

$\star * \star *$

rosette
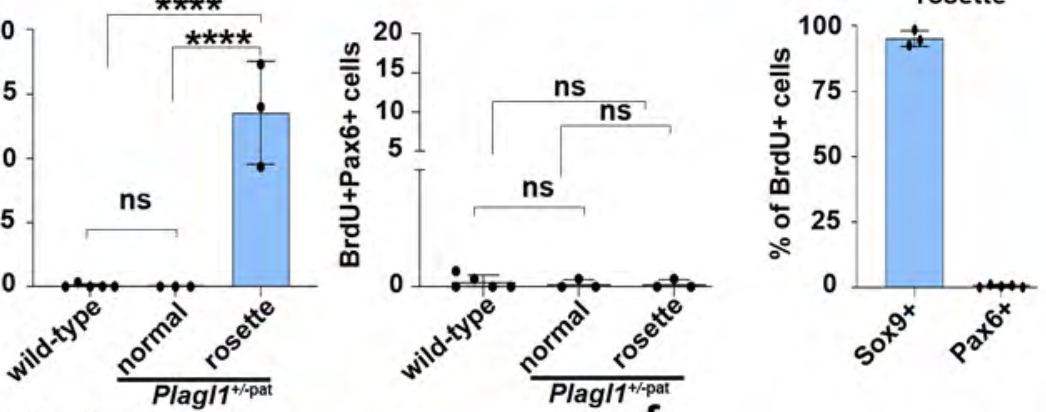

BrdU birthdating P7 $\rightarrow$ P14

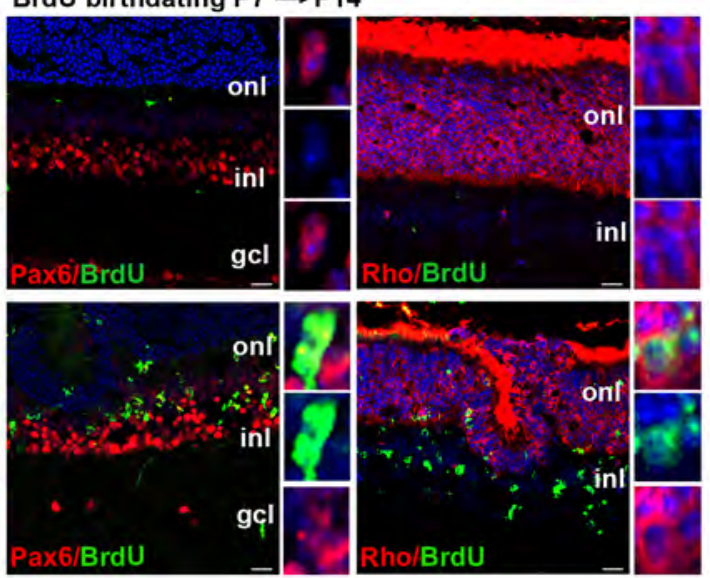

Plagl1 ${ }^{\text {t/pat }} \square$






\section{a}
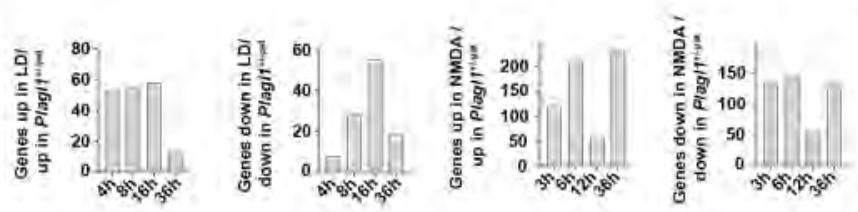

b
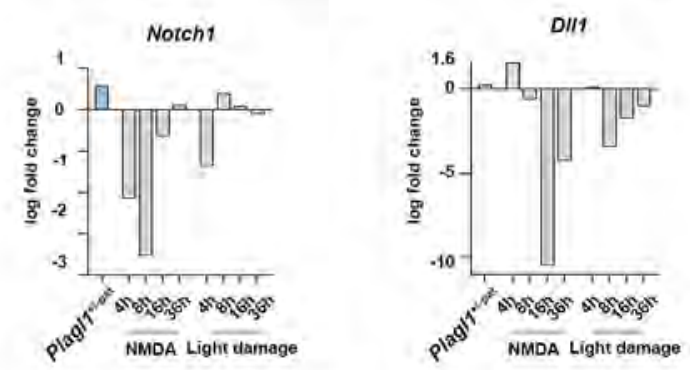

C
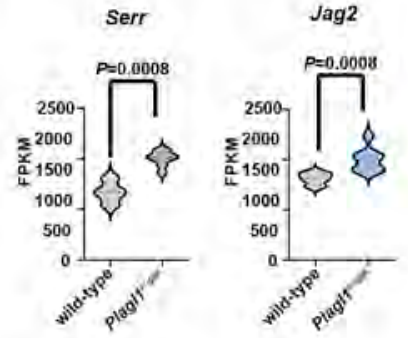

d
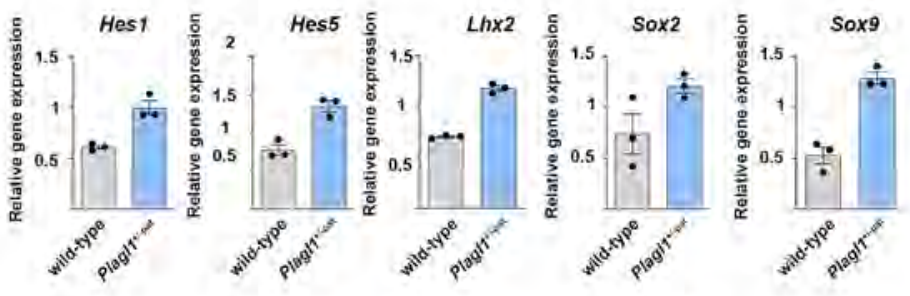

e


Lhx2
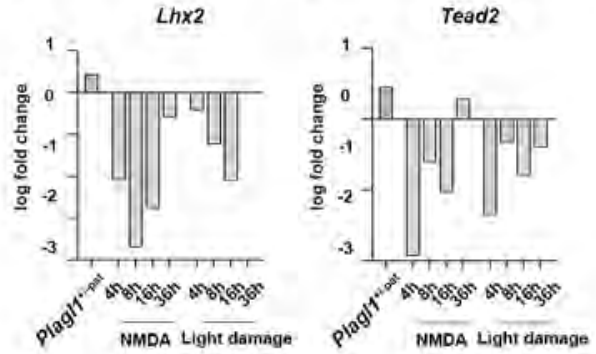
a $\quad$ Cep131

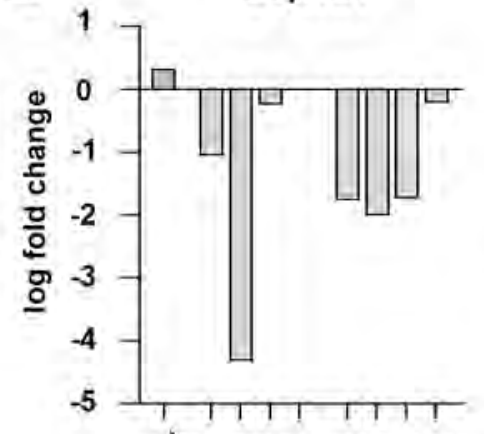

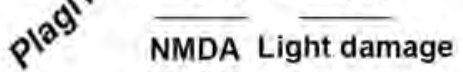

b
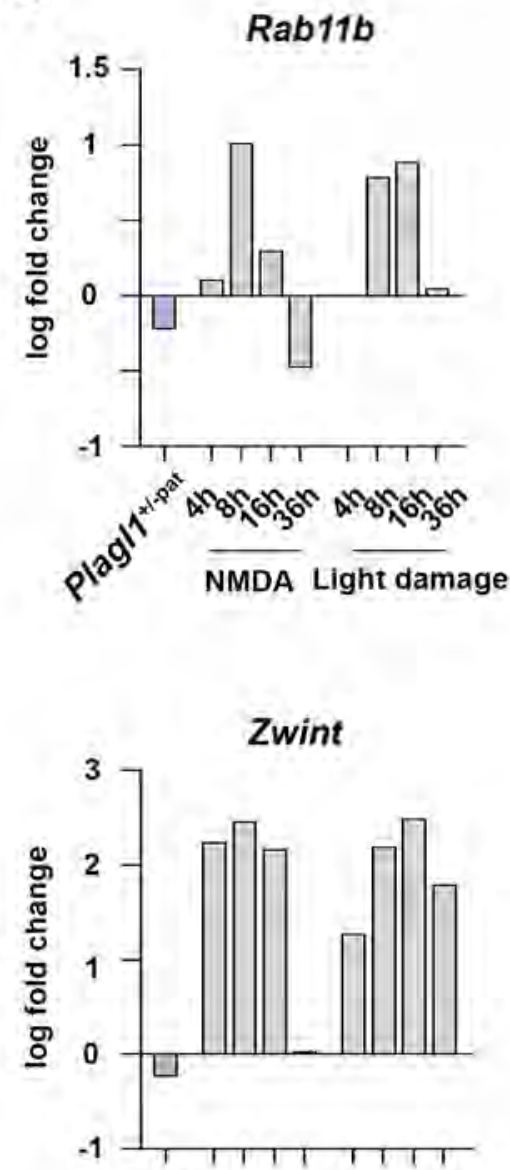

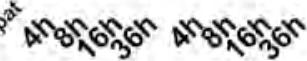

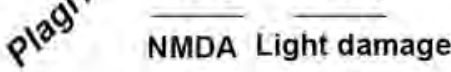

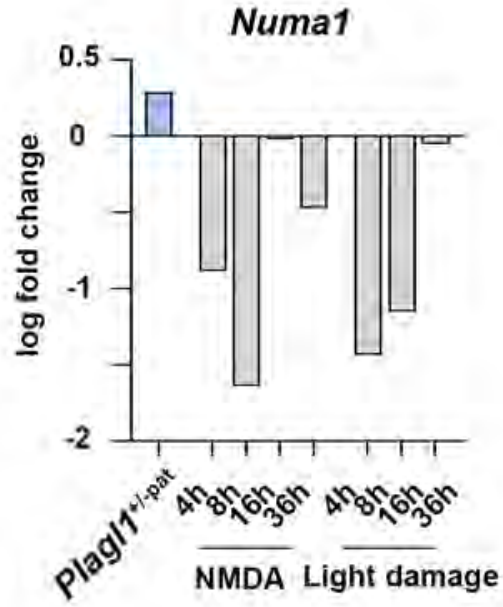

Rps3
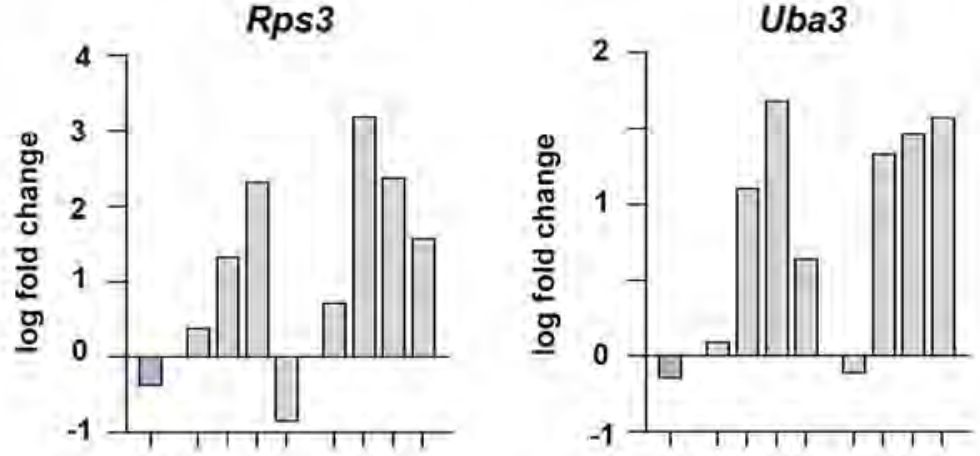

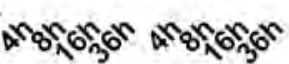

NMDA Light damage

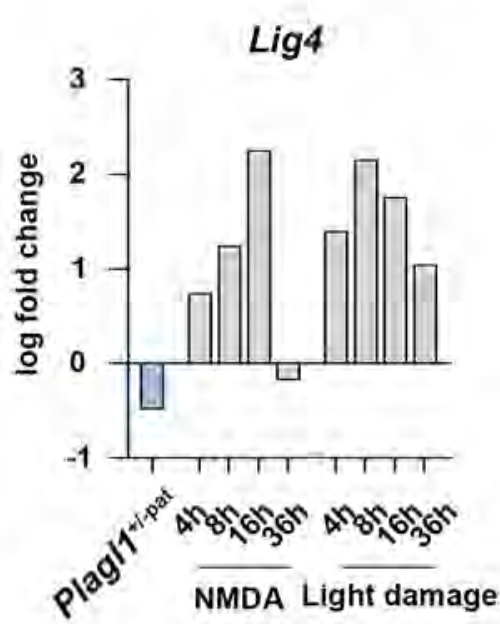

Touahri et al. Suppl. Fig. 6 